\title{
Some Results on Odd Astral Configurations
}

\author{
Leah Wrenn Berman \\ Department of Mathematics and Computer Science \\ Ursinus College, Collegeville, PA, USA \\ Iberman@ursinus.edu
}

\begin{abstract}
Submitted: Aug 15, 2004; Accepted: Mar 23, 2006; Published: Mar 30, 2006
\end{abstract}
Mathematics Subject Classification: 51A20, 52C35

\begin{abstract}
An astral configuration $\left(p_{q}, n_{k}\right)$ is a collection of $p$ points and $n$ straight lines in the Euclidean plane where every point has $q$ straight lines passing through it and every line has $k$ points lying on it, with precisely $\left\lfloor\frac{q+1}{2}\right\rfloor$ symmetry classes (transitivity classes) of lines and $\left\lfloor\frac{k+1}{2}\right\rfloor$ symmetry classes of points. An odd astral configuration is an astral configuration where at least one of $q$ and $k$ is odd. This paper presents all known results in the classification of odd astral configurations where $q$ and $k$ are both at least 4 .
\end{abstract}

\section{Introduction}

There are two main kinds of objects which are referred to as $\left(p_{q}, n_{k}\right)$ configurations. The first kind is a combinatorial configuration, which is a set of $p$ objects, called "points", and $n$ collections of "points", called "lines", so that each "point" is contained in $q$ of the "lines" and each "line" contains $k$ of the "points". Combinatorial configurations have been studied extensively since the mid-1800's; for modern investigations, see [8], [5] and [13]. The second kind of configuration is a geometric configuration. The "points" of the combinatorial configuration become actual points in some Euclidean space (almost always the plane), and the "lines" of the combinatorial configuration are straight lines in the Euclidean space. For the remainder of the paper, the term "configuration" refers to a geometric configuration, and all configurations will be in the Euclidean plane.

In [3] and [2], the author presented results on a particular variety of highly symmetric geometric configurations, known as astral configurations, where $q$ and $k$ are both even. The current work extends those results to some cases where $q$ or $k$ is odd and $q$ and $k$ are both at least 4; for an example of such a configuration, see Figure 1. Astral configurations initially were introduced in [9] and [10]; recently, astral configurations have been discussed 
as a special case of the more general notion of polycyclic configurations in [6]. Unless otherwise stated, in figures in this paper, objects with the same color are members of the same symmetry class.

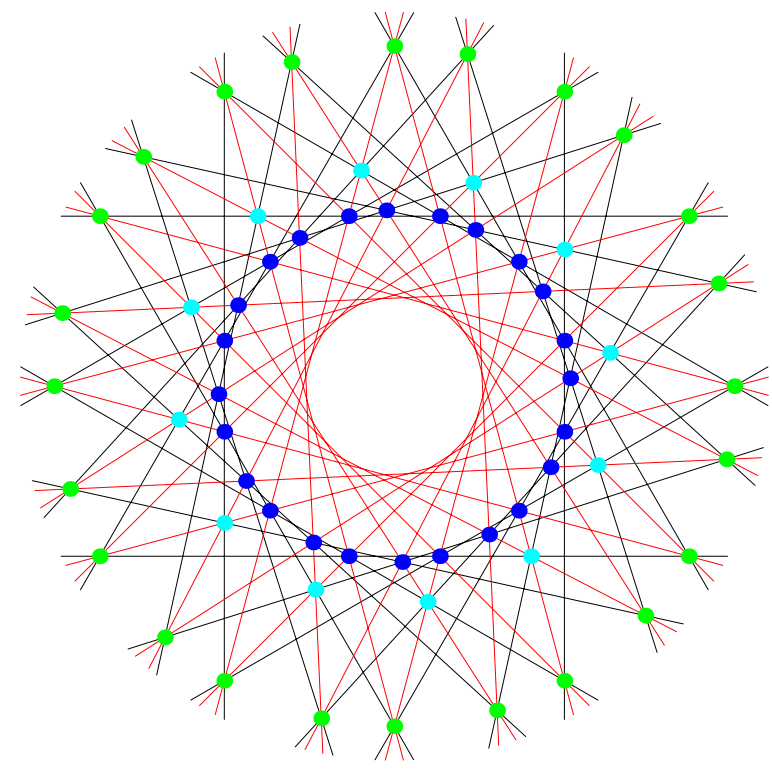

Figure 1: An astral configuration $\left(60_{4}, 48_{5}\right)$

\section{Definitions and preliminary lemmas}

The following definitions and lemmas were presented in [2] (some have been restated slightly). They are repeated here for clarity; all proofs may be found in [2].

An astral configuration $\left(p_{q}, n_{k}\right)$ is a collection of $p$ points and $n$ straight lines in the Euclidean plane with the following properties:

1. every point lies on $q$ lines;

2. every line passes through $k$ points;

3. there are precisely $\left\lfloor\frac{q+1}{2}\right\rfloor$ symmetry classes of lines;

4. there are precisely $\left\lfloor\frac{k+1}{2}\right\rfloor$ symmetry classes of points.

The symmetry classes of points or lines are precisely the transitivity classes of the points or lines under the rotations and reflections of the plane that map the configuration to itself. Note that in a $\left(p_{q}, n_{k}\right)$ configuration, if a straight line in the plane has $k$ points on it, at most two of the points can be in the same symmetry class, and similarly with the 
lines, since two lines can intersect only at a single point. Therefore, $\left\lfloor\frac{k+1}{2}\right\rfloor$ (respectively, $\left\lfloor\frac{q+1}{2}\right\rfloor$ ) is the fewest number of symmetry classes of points (respectively, lines), and so the most symmetry, that a configuration can have. Thus, the term astral configuration refers to the kind of geometric configurations which have as much symmetry as possible.

A $\left(p_{q}, n_{k}\right)$ configuration is called a configuration of class $[q, k]$, or, usually, a $[q, k]$ configuration, when one is interested in emphasizing the kinds of incidences, rather than in how many points and lines there are in the configuration. An astral configuration of class $[q, k]$ is called odd if at least one of $q$ or $k$ is odd; if both $q$ and $k$ are even, the configuration is called even. Even astral configurations are completely classified in [2]. Most of the results in [2] and this paper originally appeared in the author's Ph.D. thesis, $[4]$.

In an astral configuration with $k$ points incident with each line, where $k$ is odd, points on a given line may be partitioned into pairs by symmetry class, leaving one point that is not paired up. The collection of all such "leftover" points forms a symmetry class, and this symmetry class of points is called the special symmetry class. For all other symmetry classes of points, there will be exactly two points in the symmetry class incident with each line. Similarly, in an astral $[q, k]$ configuration with $k$ odd, the special class of lines is the single symmetry class of lines with exactly one line from the class incident with each point.

Astral configurations come in two varieties. An astral $[q, k]$ configuration of type 1 satisfies the condition that the set of points in every symmetry class of points in the configuration forms the vertices of a regular polygon; such a configuration will be abbreviated as $[q, k]^{1}$. For an example of an astral $[4,5]^{1}$ configuration, see Figure 2. In an astral type 2 configuration, there is some symmetry class of points which does not form the vertices of a regular polygon; astral type 2 configurations will be abbreviated as $[q, k]^{2}$. For example, neither the outer nor the inner ring of points in Figure 1, which is an astral $[4,5]^{2}$ configuration, forms the vertices of a regular polygon, although the middle ring does.

The size of a type 1 configuration is the cardinality of the largest symmetry class of points that forms the vertices of a regular polygon. Note that the size of the configuration given in Figure 2 is 30, although the special class of points has only 15 points in it. If a type 1 configuration is of size $m$ and it does not have the symmetries of a regular $m$-gon, then it must have the symmetries of a regular $\frac{m}{2}$-gon; the configuration in Figure 2 has the symmetries of a 15-gon. Note that every even configuration of size $m$ has the symmetries of a regular $m$-gon.

\subsection{Multiples of a configuration}

Beginning with a type 1 astral configuration of size $m$ with the symmetries of a regular $m$-gon, additional type 1 configurations may be formed by adding $r-1$ equally-spaced concentric copies of the original configuration - i.e., the new configuration will have the 


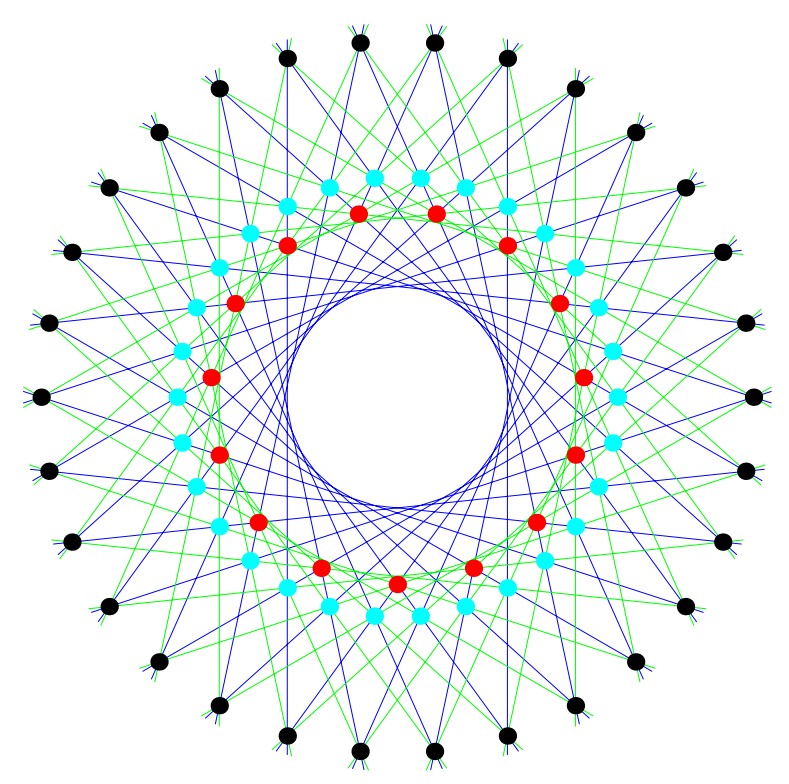

Figure 2: An astral $[4,5]^{1}$ configuration of size 30

$j^{\text {th }}$ copy rotated by $\frac{2 j \pi}{m r}$ radians. This new configuration is called an r-multiple, or, more simply, a multiple of the original configuration.

In addition, taking two copies of a size $m$ type 1 configuration, rotating one through any angle $\alpha$ which is not an integer multiple of $\frac{\pi}{m}$, and placing it concentrically on the first one yields a type 2 astral configuration; that such a configuration is astral has been shown in Lemma 2.1 (proved in [2]). The type 2 configurations produced from this process are called ordinary type 2 configurations; other type 2 configurations are called extraordinary.

Lemma 2.1. Ordinary $[q, k]^{2}$ configurations are astral.

\subsection{Diametral points}

Label the vertices of an $m$-gon consecutively as $v_{0}, \ldots, v_{m-1}$. A diagonal of the $m$-gon is of span $c$ if it connects vertices $v_{i}$ and $v_{i+c}$, where indices are taken modulo $m$ (and in general, $1 \leq c \leq m / 2$ ). In Figure 2, the green lines may be viewed as diagonals of the outer 30-gon of span 10 and the blue lines as diagonals of span 12. Given a regular polygon and a diagonal of span $c$, label the intersection points of the diagonal with other span $c$ diagonals as $c_{1}, c_{2}, \ldots, c_{\left\lfloor\frac{m}{2}\right\rfloor}$, counted from the midpoint of the diagonal and travelling in one direction (usually, to the left). Considering the set of points with symbol $c_{i}$, if $i>c$, the point is outside the polygon, if $i=c$ the point is a vertex of the polygon, and if $i<c$ the point is interior to the polygon; see Figure 3. Also, the point with symbol $c_{-d}$ is the $d$-th intersection point along the span $c$ diagonal counted in the other direction.

A line is diametral with respect to a regular convex $m$-gon if it passes through the 


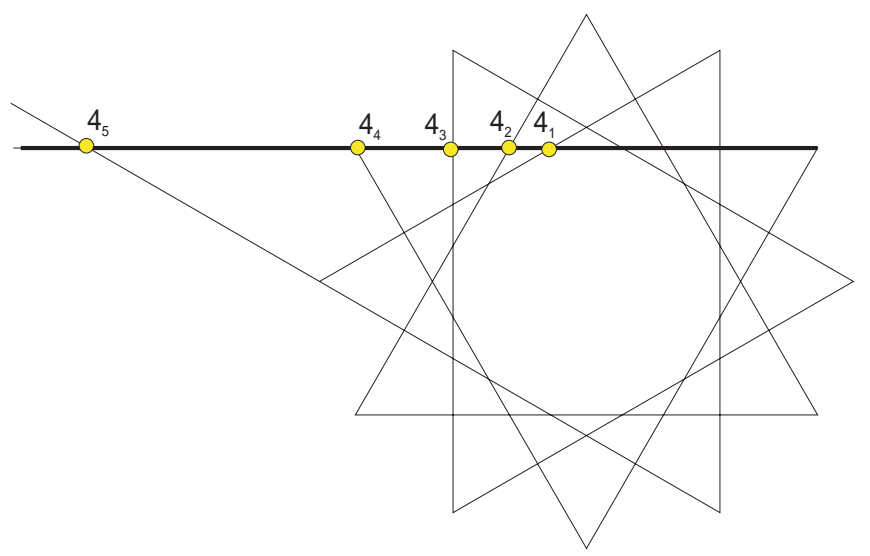

Figure 3: Examples of the symbols $c_{d}$; in this example, $c=4$.

center of the $m$-gon and one of the vertices of the polygon. Note that if $m$ is even, diametral lines correspond to the ordinary notion of diameters of a regular polygon, i.e, they pass through two vertices and the center of the polygon and are lines of span $\frac{m}{2}$. A line in a type 1 configuration is diametral if it is diametral for the underlying regular polygon formed by the ring of vertices that is farthest from the center of the configuration. A line in a configuration is semidiametral if it passes through the center of the $m$-gon and is the angle bisector of two diametral lines. A point is diametral if it lies on a diametral line, and a point is semidiametral if it lies on a semidiametral line.

Lemma 2.2. Choose a span $c$ diagonal of a regular, convex m-gon, and label the intersection points of the diagonal with other span $c$ diagonals as $c_{1}, c_{2}, \ldots, c_{c}, \ldots, c_{\left\lfloor\frac{m}{2}\right\rfloor}$. If $m$ is even, the intersection points $c_{i}$ which are diametral are precisely those for which the parity of $c$ and $i$ is the same, and the other intersection points are semidiametral. If $m$ is odd, all points $c_{i}$ are diametral.

\subsection{Polars}

Given an astral $[q, k]$ configuration in the extended Euclidean plane (that is, the Euclidean plane with the line at infinity appended), a new $[k, q]$ configuration may be constructed by taking the polar of the configuration with respect to a circle concentric with the configuration. The resulting configuration is astral in the ordinary Euclidean plane as long as the original configuration contained no lines passing through the center of the configuration. Taking polars is an easy way to produce new configurations from previously classified ones. 


\section{Theorems about even configurations}

If only one of $q$ or $k$ is even, then odd astral configurations may be constructed using even astral configurations. To see this, suppose that an astral $[4,5]$ configuration exists. It must have three symmetry classes of points, one of which is the special class. Ignore the special class of points, and the resulting configuration is an astral $[4,4]$ configuration. Thus, if the $[4,5]$ configuration is to exist, it must be constructed using astral $[4,4]$ configurations, and similarly for other odd configurations.

Hence, to prove results about $[2 s, 2 t+1]$ and $[2 s+1,2 t]$ configurations, it is useful to know results about the existence and nonexistence of various even astral configurations. The following results were discussed and proved in [2] and [3].

A $[4,4]^{1}$ configuration of size $m$ consists of two concentric $m$-gons corresponding to the two symmetry classes of points. It is denoted as $m \# a_{b} c_{d}$, where $a$ and $c$ are the spans of diagonals of the $m$-gons corresponding to lines of the configuration. Since any $[4,4]$ configuration must have four lines passing through each point and only four points on each line, $b$ and $d$ must be chosen so that $a_{b}$ and $c_{d}$ are the same point of the configuration.

Theorem 3.1. All $[4,4]^{1}$ configurations are listed in the following: there are two infinite families, $(6 k) \#(3 k-j)_{3 k-2 j}(2 k)_{j}$ for $j=1, \ldots, 2 k-1, k>1, j \neq k$ and $j \neq \frac{3 k}{2}$, and $(6 k) \#(3 k-2 j)_{j}(3 k-j)_{2 k}$, for $k>1, j=1, \ldots, k-1$. There are 27 connected sporadic configurations, with $m=30,42$, and 60, listed in Table 1, where a configuration is sporadic if it is not a member of one of the infinite families. Finally, there are multiples of the sporadic configurations.

$m=30$

$$
\begin{array}{lll}
30 \# 4_{1} 7_{6} & 30 \# 6_{1} 7_{4} & 30 \# 6_{1} 11_{10} \\
30 \# 6_{2} 8_{6} & 30 \# 7_{2} 12_{11} & 30 \# 8_{1} 13_{12} \\
30 \# 10_{1} 11_{6} & 30 \# 10_{6} 12_{10} & 30 \# 10_{7} 13_{12} \\
30 \# 11_{2} 12_{7} & 30 \# 11_{6} 14_{13} & 30 \# 12_{1} 13_{8} \\
30 \# 12_{4} 14_{12} & 30 \# 12_{7} 13_{10} & 30 \# 13_{6} 14_{11}
\end{array}
$$

$m=42$

$$
\begin{array}{lll}
42 \# 6_{1} 13_{12} & 42 \# 11_{6} 18_{17} & 42 \# 12_{1} 13_{6} \\
42 \# 12_{5} 19_{18} & 42 \# 17_{6} 18_{11} & 42 \# 18_{5} 19_{12}
\end{array}
$$

$m=60$

$$
\begin{array}{lll}
60 \# 9_{2} 22_{21} & 60 \# 12_{5} 2_{24} & 60 \# 14_{3} 27_{26} \\
60 \# 21_{2} 22_{9} & 60 \# 24_{5} 25_{12} & 60 \# 26_{3} 27_{14}
\end{array}
$$

Table 1: The sporadic astral $[4,4]^{1}$ configurations 
In addition, $[4,4]^{2}$ configurations were classified in the following (slightly restated from [3]):

Theorem 3.2. All $[4,4]^{2}$ configurations are ordinary.

The proof of Theorem 3.1 was the main content of [3].

Proposition 3.3. Every $[2 s, 2 t]^{2}$ configuration is ordinary.

Following the notation introduced for astral $[4,4]^{1}$ configurations, an astral $[6,4]^{1}$ configuration is denoted by $m \# a_{b} c_{d} z_{w}$, where $a_{b}, c_{d}$, and $z_{w}$ represent the same point of the configuration.

Theorem 3.4. These are all the astral [6,4] configurations: the type 1 configurations $30 \# 8_{1} 10_{7} 13_{12}, 30 \# 6_{1} 7_{4} 11_{10}, 30 \# 11_{2} 12_{7} 13_{10}, 30 \# 9_{3} 10_{6} 12_{10}, 30 \# 10_{1} 11_{6} 14_{13}$, multiples of these, and ordinary type 2 configurations formed from the already-listed configurations.

Every astral [4,6] configuration is formed as the polar of an astral [6, 4] configuration, but it is convenient to list them separately. An astral $[4,6]$ configuration has two symmetry classes of lines and three symmetry classes of points. If it is formed from two astral $[4,4]^{1}$ configurations $m \# a_{b} c_{d}$ and $m \# a_{e} c_{f}$, it will be denoted $m \#\left(a_{b} c_{d}\right)\left(a_{e} c_{f}\right)$, where each set of symbols enclosed in parentheses represent one of the intersection points of the $a$ and $c$ diagonals. That is, $a_{b}$ and $c_{d}$ represent the same point in the configuration, as do $a_{e}$ and $c_{f}$.

Proposition 3.5. The astral $[4,6]$ configurations are the following: $30 \#\left(12_{1} 1_{8}\right)\left(12_{7} 13_{10}\right), 30 \#\left(10_{4} 11_{7}\right)\left(10_{1} 11_{6}\right), 30 \#\left(10_{2} 13_{11}\right)\left(10_{7} 13_{12}\right)$, $30 \#\left(10_{3} 12_{9}\right)\left(10_{6} 12_{10}\right), 30 \#\left(13_{1} 14_{10}\right)\left(13_{6} 14_{11}\right)$, and their multiples, plus ordinary type 2 configurations formed from these.

Theorem 3.6. No astral configurations $[2 s, 2 t]$ exist where $s$ and $t \geq 3$. Moreover, for $s \geq 2$ and $t \geq 4$, there are no astral $[2 s, 2 t]$ and $[2 t, 2 s]$ configurations.

Lemma 3.7. If no astral $[2 s, 2 t]$ configuration exists, then no astral $[2 s+x, 2 t+y]$ configuration exists either, where $x, y=0,1,2, \ldots$

\section{General results for $[2 s, 2 t+1]^{1}$ and $[2 s+1,2 t]^{1}$ config- urations}

Suppose an astral $[2 s, 2 t+1]^{1}$ configuration exists; it has $s$ line spans, called $a_{1}, \ldots, a_{s}$. Since $2 t+1$ is odd, one of the symmetry classes of points is special. Removing this special class of points yields an astral $[2 s, 2 t]^{1}$ configuration. Consider one of the special points. Since it lies on a $2 s$-diagonal intersection, in particular, it it lies on some span $a_{1}$ line and must be the $e$-th intersection point of that line with another $\operatorname{span} a_{1}$ line, 
counted from the midpoint in some direction; without loss of generality, it is to the left of the midpoint. As usual, this point has symbol $\left(a_{1}\right)_{e}$. The symmetry of the underlying $[2 s, 2 t]^{1}$ configuration forces that the $e$-th intersection point to the right of the midpoint also participates in a $2 s$-diagonal intersection, since the underlying $[2 s, 2 t]^{1}$ configuration has as one of its symmetries the mirror which passes through the center of the configuration and the midpoint of the given span $a_{1}$ line. Adding to the $[2 s, 2 t]^{1}$ configuration both of the possible points with symbol $\left(a_{1}\right)_{e}$ on each line yields an astral $[2 s, 2 t+2]^{1}$ configuration. This discussion is summarized in the following lemma:

Lemma 4.1. If an astral $[2 s, 2 t+1]^{1}$ configuration exists, then an astral $[2 s, 2 t+2]^{1}$ configuration must also exist. Hence, if no astral $[2 s, 2 t+2]^{1}$ configuration exists, then no astral $[2 s, 2 t+1]^{1}$ configuration exists, either.

Corollary 4.2. If an astral $[2 t+1,2 s]^{1}$ configuration exists which does not use diameters, then an astral $[2 t+2,2 s]^{1}$ configuration must also exist. Hence, if no astral $[2 t+2,2 s]^{1}$ configuration exists, then if an astral $[2 t+1,2 s]^{1}$ configuration exists, it must be constructed by adding diameters to a $[2 t, 2 s]^{1}$ astral configuration.

Proof. This follows from Lemma 4.1 by polarity.

\section{Astral $[4,5]^{1}$ and $[5,4]^{1}$ configurations}

\subsection{Astral $[4,5]^{1}$ configurations}

A $[4,5]^{1}$ configuration may be constructed by adding a class of points appropriately to a $[4,4]^{1}$ configuration that has additional intersections of four diagonals. Astral $[4,6]^{1}$ configurations have the appropriate intersections, but they have 6 points on a line instead of five.

If an astral $[4,5]^{1}$ configuration exists, Lemma 4.1 implies that it is constructed from a $[4,6]^{1}$ configuration. Moreover, each line in the $[4,5]^{1}$ configuration must contain only one point from the special class of points. Let $S$ be the name of the symmetry class of points in the $[4,6]^{1}$ configuration from which the special class of points is formed in the $[4,5]^{1}$ configuration, and call the special class of points in the $[4,5]^{1}$ configuration $\hat{S}$. The symmetries of the $[4,5]^{1}$ configuration must act transitively on $\hat{S}$, so locally, any point

in $\hat{S}$ looks like any other point in $\hat{S}$. Imagine that the points used for $\hat{S}$ are colored black, and the points in $S \backslash \hat{S}$ are colored red. Every line in the $[4,5]^{1}$ configuration must contain one red point and one black point, so that it has five points on it rather than six, so exactly half the points of $S$ are used to form $\hat{S}$.

Note that the points of $S$ are concyclic. Say that two points in $S$ are neighbors if they are adjacent to each other viewed as points on the circle. In the situation of Figure 4 , note that every red vertex has two black neighbors, and vice versa. 


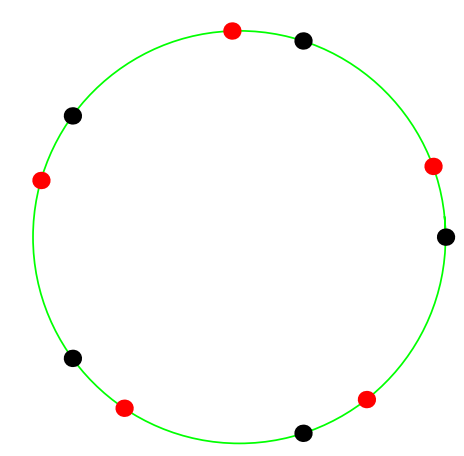

Figure 4: A schematic of the symmetry class of points which includes the special class of points, viewed on a circle to determine neighbors.

Since any point in the special class must look like any other, in particular, if one black (special) point has a red neighbor, then all black points must have a red neighbor. Therefore, travelling along the circle which passes through $S$, there are precisely two possibilities: the pattern of special points within $S$ is either two points in the special class alternating with two points not in the symmetry class, so that each black point has a black neighbor and a red neighbor, or every other point is in the special class of points, so that each black point has only red neighbors.

Consider the case where the pattern of neighbors is two points in the special class followed by two points not in the special class. For this pattern to be possible, the size of the configuration, $m$, is congruent to $0 \bmod 4$. If $m \equiv 0 \bmod 4$, it is necessary that $q \equiv 0$ mod 2 , since $m=6 \cdot 5 q$. In this case, by Lemma 2.2, every point of the configuration lies on a diameter of the configuration. Given the constraints of the special class of points, the available symmetries are mirrors which pass through the center of the configuration at an angle halfway between two special points (so that every other semidiametral mirror in the $[4,6]$ configuration is a symmetry) and rotations about the center with angle $\frac{4 \pi}{m}$. When applied to the configuration, these symmetries will map any point in the special class of points to any other point in that class. Unfortunately, they will have the same effect on points in the other symmetry classes of the $[4,6]$ configuration - if points in the orbit are colored red and the other points which were in the same symmetry class viewed in the $[4,6]$ configuration are colored black, then travelling along the circle which passes through the symmetry class of points of the $[4,6]$ configuration under consideration generates the pattern of two red points followed by two black points, etc. Thus, where once there were three symmetry classes of points, now there are five symmetry classes, so the resulting $[4,6]$ configuration is not astral.

In the case of using every other point, either the points in the special class are on diameters or they are not. If the special class of points is formed from the only symmetry class in the $[4,6]$ configuration which is not diametral, then the available symmetries are the semidiametral mirrors and rotations through $\frac{2 \pi}{m}$, and these two kinds of symmetries suffice to map any point in a non-special symmetry class to any other point in that class. 
If in the $[4,6]$ configuration all three symmetry classes are diametral or if two of them are non-diametral, applying the symmetries will cause the other symmetry class which matches the special class (diametral if the special class is diametral, non-diametral if the special class is non-diametral) to be partitioned into two orbits which interlace so that every other point is in one orbit, breaking astrality.

Thus, $[4,5]^{1}$ configurations may only be constructed from $[4,6]^{1}$ configurations which have one symmetry class of points which is not diametral and two which are, and they must be constructed by taking every other point from the non-diametral symmetry class. In particular, $q$ must be odd. So, from Proposition 3.5, the available $[4,6]$ configurations are: $q \cdot 30 \#\left(10_{4} 11_{7}\right)\left(10_{1} 11_{6}\right), q \cdot 30 \#\left(10_{2} 13_{11}\right)\left(10_{7} 13_{12}\right), q \cdot 30 \#\left(10_{3} 12_{9}\right)\left(10_{6} 12_{10}\right)$, and $q \cdot 30 \#\left(13_{1} 14_{10}\right)\left(13_{6} 14_{11}\right)$, where $q$ is odd.

By $m \#\left(a_{b} c_{d}\right)\left(a_{e} c_{f}\right)^{*}$ denote the configuration which has vertices with symbols $\left(a_{a}\right)_{i}=$ $\left(c_{c}\right)_{i},\left(a_{b}\right)_{i}=\left(c_{d}\right)_{i}$ for all $i$ and $\left(a_{e}\right)_{i}=\left(c_{f}\right)_{i}$ for $i=0,2,4, \ldots, m-2$ (so that every other vertex in the $a_{e}$ ring is used).

Theorem 5.1. The only astral $[4,5]^{1}$ configurations are $(30 q) \#\left((10 q)_{(6 q)}(12 q)_{(10 q)}\right)\left((10 q)_{(3 q)}(12 q)_{(9 q)}\right)^{*}$, where $q$ is odd.

Proof. Given a $[4,5]^{1}$ configuration $m \#\left(a_{b} c_{d}\right)\left(a_{e} c_{f}\right)^{*}$, label the vertices with symbol $a_{a}$ as $v_{i}$, the vertices with symbol $a_{b}=c_{d}$ as $w_{i}$, and the vertices $a_{e}=c_{f}$ as $u_{i}$. Specifically,

$$
u_{i}=\frac{\cos \left(\frac{a \pi}{m}\right)}{\cos \left(\frac{e \pi}{m}\right)}\left(\cos \left(\frac{\pi}{m}(2 i+a+e)\right), \sin \left(\frac{\pi}{m}(2 i+a+e)\right)\right) \text {, }
$$

and note that only the points $u_{i}$ for $i=0,2,4, \ldots, m-2$ are used in the configuration $m \#\left(a_{b} c_{d}\right)\left(a_{e} c_{f}\right)^{*}$.

Note that the configuration $q \cdot\left(m \#\left(a_{b} c_{d}\right)\left(a_{e} c_{f}\right)^{*}\right)$ is not the same configuration as $(m q) \#\left((a q)_{(b q)}(c q)_{(d q)}\right)\left((a q)_{(e q)}(c q)_{(f q)}\right)^{*}$; the first configuration has the special class of lines passing through $v_{i}$ and $v_{i+e}$ for all $i=1,2, \ldots, q$.

A configuration $m \#\left(a_{b} c_{d}\right)\left(a_{e} c_{f}\right)^{*}$ has five points lying on every line with symbol $a_{e}=c_{f}$ precisely when $e$ and $f$ are both odd. To see this, consider a span $a$ diagonal. By definition, it passes through points $v_{i}$ and $v_{i+a}$ for some choice of $i=0,1,2, \ldots, m-1$. It also passes through points with symbol $a_{b}$; in particular, for some $j=0,1,2, \ldots, m-1$ it passes through points $w_{j}$ and $w_{j+b}$, since the span $a$ diagonals of the $v_{i}$ vertices are span $b$ diagonals when viewed as diagonals of the $w_{j}$ vertices. Similarly, for the points $u_{k}$ with symbol $a_{e}$, the span $a$ diagonals are span $e$ diagonals when viewed as the diagonals of the points $u_{k}$ for $k=0,1,2, \ldots, m-1$. Note that only the points $u_{k}$ with $k$ even are points of the possible configuration.

Thus, the span $a$ diagonal passes through the points $v_{i}, v_{i+a}, w_{j}, w_{j+b}, u_{k}$ and $u_{k+e}$. However, sometimes $u_{k}$ and $u_{k+e}$ are points of the configuration and sometimes they are not; we are interested in the case when for any choice of $k$, one is and one isn't. But this occurs precisely when $e$ is odd. To see this, note if $e$ is odd and $k$ is even, $e+k$ is odd, 
so $u_{k}$ is a point of the configuration and $u_{k+e}$ is not, while if $e$ and $k$ are both odd (so that $e+k$ is even), then $u_{k+e}$ is a point of the configuration and $u_{k}$ is not. Thus, if $e$ is odd, all span $a$ diagonals contain five points of the configuration. On the other hand, if $e$ is even and $k$ is odd, then neither $u_{k}$ nor $u_{k+e}$ are points of the configuration, so the span $a$ diagonal contains only four points of the configuration, while if $e$ and $k$ are both even, both $u_{k}$ and $u_{k+e}$ are points of the configuration, so the span $a$ diagonal contains six points of the configuration. Thus, if $e$ is even, half the span $a$ diagonals contain six points and the other half contain only four points of the configuration.

Similarly, if $f$ is odd, the span $c$ diagonals will contain precisely five points of the configuration, while if $f$ is even, half the diagonals will contain six points and the other half will contain only four points.

There is exactly one infinite family of configurations $m \#\left(a_{b} c_{d}\right)\left(a_{e} c_{f}\right)^{*}$ with both $e$ and $f$ odd, namely, $(30 q) \#\left((10 q)_{(6 q)}(12 q)_{(10 q)}\right)\left((10 q)_{(3 q)}(12 q)_{(9 q)}\right)^{*}$, for $q$ any odd number.

\subsection{Astral $[5,4]^{1}$ configurations}

Since none of the configurations listed in Theorem 5.1 contains diagonals which pass through the origin, their polars with respect to a concentric circle will be astral $[5,4]^{1}$ configurations. Note that these astral $[5,4]^{1}$ configurations may be written as

$$
(30 q) \#(10 q)_{(6 q)}(12 q)_{(10 q)}(3 q)_{(9 q)} *
$$

with $q$ odd, where for the symmetry class of lines in the polar corresponding to the special class of points in the original configuration (marked with * in the configuration symbol), every other line is used, so that in the polar configuration, for the special class of lines, for example, only the left-hand line is used. Figure 5 is an astral $[5,4]^{1}$ configuration constructed as the polar of the configuration shown in Figure 2.

However, there are additional astral $[5,4]^{1}$ configurations which may be obtained by adding diameters to appropriate astral $[4,4]^{1}$ configurations, as in Figure 6 ; these may be determined by applying Lemma 2.2. Diameters may be added to a configuration $m \# a_{b} c_{d}$ to form an astral $[5,4]^{1}$ configuration precisely when $a \equiv b \bmod 2$ (and, since $a_{b}$ and $c_{d}$ are the same points, $c \equiv d \bmod 2$ as well); these will be denoted as $m \# a_{b} c_{d}, D$.

Using Lemma 2.2 and the above remark, the following is proved:

Theorem 5.2. Diameters may be added to the following astral $[4,4]$ configurations to yield astral $[5,4]^{1}$ configurations:

1. $(2 t) \cdot m \# a_{b} c_{d}$ for any astral configuration $m \# a_{b} c_{d}$;

2. $(6 k) \#(3 k-j)_{(3 k-2 j)} 2 k_{j}$, if $j$ is even and $k$ is odd;

3. $(6 k) \#(3 k-2 j)_{j} 3 k-j_{2 k}$, if $j$ and $k$ are both odd; 


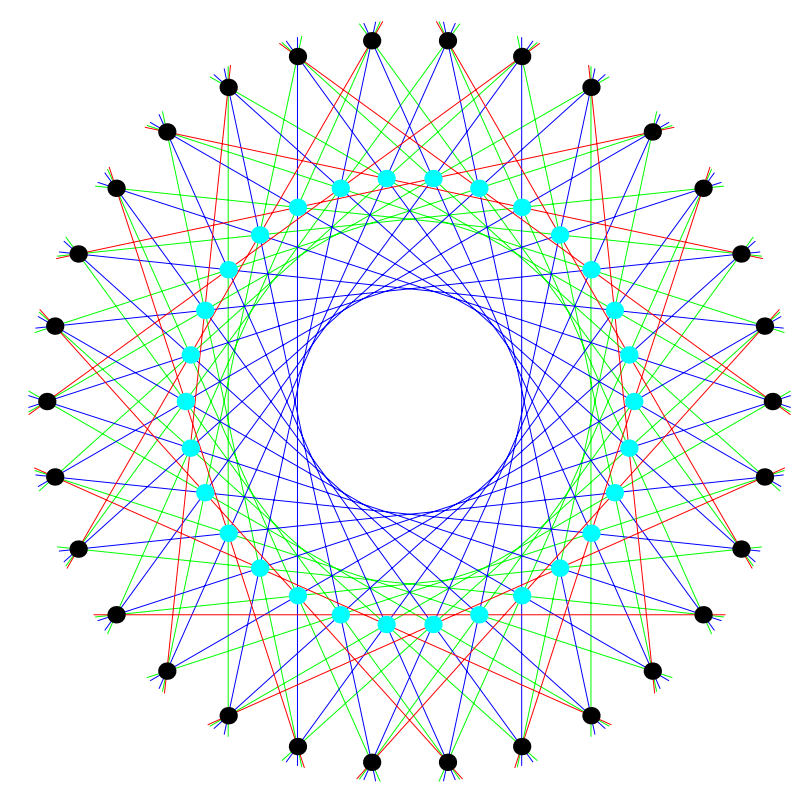

Figure 5: The astral $[5,4]^{1}$ configuration $30 \# 10_{6} 12_{10} 9_{3}^{*}$

4. $q \cdot 30 \# 6_{2} 8_{6}, q \cdot 30 \# 10_{6} 12_{10}, q \cdot 30 \# 12_{4} 14_{12}$ for any odd $q$.

Recall from Proposition 3.6 that no astral configurations of class $[q, k]$ exist if one of $q$ or $k$ is at least 8 and the other is at least 4 . In particular, there are no astral configurations $[4,8],[8,4]$, or $[5,8]$.

Corollary 5.3. There are no astral $[4,7]^{1}$ or $[6,5]^{1}$ configurations.

Proof. The nonexistence of astral $[4,7]^{1}$ configurations follows from the nonexistence of astral $[4,8]^{1}$ configurations (Theorem 3.6) and Lemma 4.1; the nonexistence of astral $[6,5]^{1}$ configurations follows from the nonexistence of astral $[6,6]^{1}$ configurations (also Theorem 3.6) and Lemma 4.1 as well.

\section{Mixed configurations}

\subsection{Extraordinary type 2 configurations}

Given an astral $[4,2 t]^{1}$ configuration of size $m$, with the two symmetry classes of lines of span $a$ and span $c$, there are many points of intersection of a single span $a$ diagonal with a single span $c$ diagonal (i.e., not an intersection point that participates in a 4-diagonal intersection); these points will be called embryonic. Choose one of them, called $x$; it is not on a mirror of symmetry of the configuration. To see this, note that each point of 


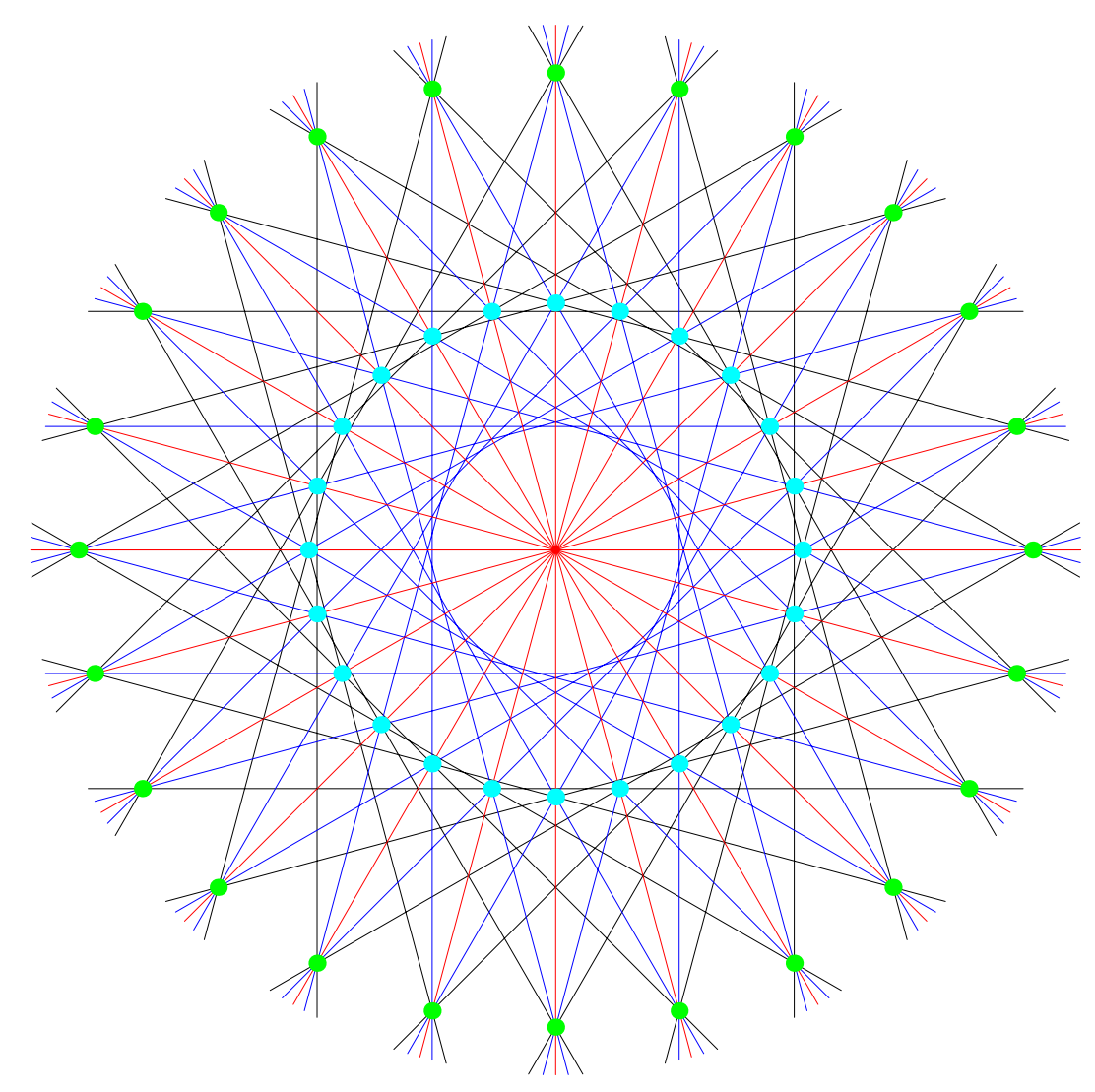

Figure 6: A $[5,4]^{1}$ configuration formed by adding diameters to the configuration $24 \# 8_{2} 10_{8}$.

intersection of a span $a$ diagonal with another span $a$ diagonal lies on one of the lines of symmetry of the $[4,2 t]^{1}$ configuration, which has the symmetries of a regular $m$-gon. For the chosen point of intersection to lie on a line of symmetry, it would also have to be part of an $a-a$ intersection, and symmetry would force it to be a 4-diagonal intersection point. But it was chosen to be the intersection of precisely two diagonals, a span $a$-diagonal and a span $c$-diagonal.

Assume the $[4,2 t]^{1}$ configuration is centered at the origin with one of its vertices located at the point $(1,0)$. Call $\alpha$ the angle formed by the ray $\langle(0,0), x\rangle$ and horizontal. Take another copy of the $[4,2 t]^{1}$ configuration and rotate it through $2 \alpha$ about the origin; color the original configuration black and the rotated configuration red. This yields a configuration with four diagonals passing through point $x$ : the black $a$ and $c$ diagonals that passed through $x$ originally and the red $a$ and $c$ diagonals from the rotated configuration. If all of the points $x$ formed in the same manner are taken as points of the configuration as well, the result is an astral $[4,2 t+1]^{1}$ configuration. A configuration constructed in this fashion will be called a mixed configuration. Figure 7 shows a mixed $[4,5]^{2}$ configuration. 


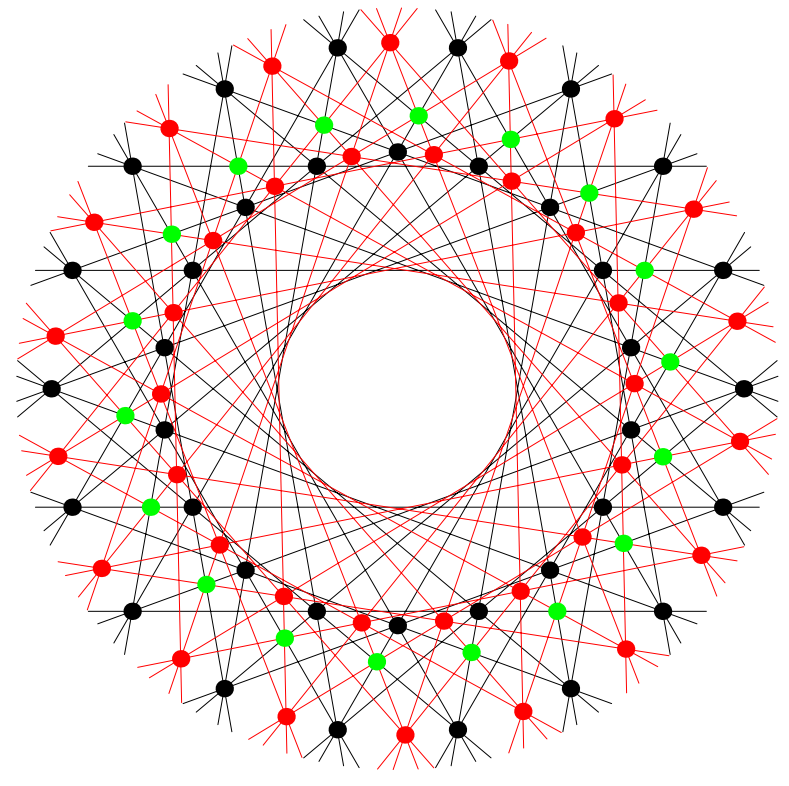

Figure 7: An astral $[4,5]^{2}$ configuration mixed from two $18 \# 6_{2} 7_{5}$ configurations, using the third $a-c$ intersection. As in the discussion, the original configuration is colored black and the rotated configuration is colored red, and the new points formed by the embryonic points are colored green.

Lemma 6.1. Mixed $[4,2 t+1]^{2}$ configurations are astral.

Proof. Call the new points formed using the embryonic points the special points of the configuration. Ignoring these special points yields an ordinary astral $[4,2 t]^{2}$ configuration. Thus, it suffices to show that the symmetries of the the ordinary $[4,2 t]^{2}$ configuration map any special point to any other special point. The symmetries of the underlying ordinary configurations were rotations by $\frac{2 \pi}{m}$ and mirrors which pass halfway between the two copies which form the ordinary configuration. The new points lie on every other mirror, so the rotations of the ordinary configuration map any special point to any other special point.

Suppose there exists a $[2 s, 2 t]^{1}$ configuration. This configuration has $s$ symmetry classes of lines; assume that the spans are $a_{1}, a_{2}, \ldots, a_{s}$. If there is a point which has exactly one line of each of the spans $a_{1}, a_{2}, \ldots, a_{s}$ passing through it, then it is called an s-embryonic point, and it can be used identically as with the embryonic points to construct an astral mixed $[2 s, 2 t+1]^{2}$ configuration.

Lemma 6.2. The only astral $[2 s, 2 t+1]^{2}$ configurations are ordinary and mixed.

Proof. Any configuration $[2 s, 2 t+1]^{2}$ has a special class of points since $2 t+1$ is odd. If the special class of points is removed, the resulting configuration is an astral $[2 s, 2 t]^{2}$ 
configuration. According to Corollary 3.3, this configuration must be ordinary - that is, it is constructed of two disjoint astral $[2 s, 2 t]^{1}$ subconfigurations. Color one of the subconfigurations black and the other subconfiguration red. Consider a point in the special class of points in the $[2 s, 2 t+1]^{2}$ configuration; it must have $2 s$ lines passing through it, two from each of the $s$ symmetry classes of lines, and suppose the $s$ symmetry classes of lines correspond to spans $a_{1}, a_{2}, \ldots, a_{s}$. By symmetry, the lines passing through the special point either are all red or all black, or there are $s$ black lines and $s$ red lines passing through it.

Case 1: Without loss of generality, say all the lines are black; then the black configuration together with the members of the special class which also have all black lines passing through them forms an astral $[2 s, 2 t+1]^{1}$ configuration, as does the red configuration with the rest of the special points, so together the black and red configurations with the special class of points forms an ordinary $[2 s, 2 t+1]^{2}$ configuration.

Case 2: There are $s$ black lines and $s$ red lines passing through the point. Since there must be two lines of each of the spans $a_{1}, a_{2}, \ldots, a_{s}$ passing through the point, symmetry forces that there must be black lines of each span $a_{1}, a_{2}, \ldots, a_{s}$ and red lines of each span $a_{1}, a_{2}, \ldots, a_{s}$ passing through the point, Thus, the black lines of span $a_{1}, a_{2}, \ldots, a_{s}$ intersect to form an $s$-embryonic point, so the $[2 s, 2 t+1]^{2}$ astral configuration may be constructed as a mixed configuration using that $s$-embryonic point.

\subsection{Explicit construction of mixed astral $[4,5]^{2}$ configurations}

There are many astral $[4,5]^{2}$ configurations; their existence is discussed in Lemma 6.2 and the preceding discussion.

Given an astral [4,4] configuration $m \# a_{b} c_{d}$, the $a-c$ intersection points may be classified. Travelling along a span $a$ diagonal beginning at the midpoint and moving to the left, one may label the $a-c$ intersections (including the ones that participate in a 4diagonal intersection) as $p_{1}, p_{2}, \ldots, p_{\left\lfloor\frac{m}{2}\right\rfloor}$, where $p_{1}$ is the first $a-c$ intersection to the left of the midpoint. Note that $p_{i}$ may be chosen where $i>a$; in this case the ring of embryonic points is farther away from the center of the configuration than the rings of configuration points with symbols $a_{a}=c_{c}$ and $a_{b}=c_{d}$. An example of this case is shown in Figure 8. Let

$$
v_{i}=\left(\cos \left(\frac{2 \pi i}{m}\right), \sin \left(\frac{2 \pi i}{m}\right)\right)
$$

and assume that the points with symbol $a_{a}=c_{c}$ are $v_{i}$ for $i=0, \ldots, m-1$. Suppose that the $a$-line under consideration is the line $\left\langle v_{i}, v_{i+a}\right\rangle$. To properly construct a mixed configuration using, say, $p_{t}$, it is first necessary to determine which line $\left\langle v_{j}, v_{j+c}\right\rangle$ for some $j$ is the $c$-line which intersects $p_{1}$.

Let $A$ be the midpoint of the line $\left\langle v_{0}, v_{a}\right\rangle$. We must determine the point (not usually of the configuration) called $v_{\gamma}$, at an angle of $\frac{2 \pi \gamma}{m}$ from horizontal on the unit circle, so that $A$ lies on $\left\langle v_{\gamma}, v_{\gamma+c}\right\rangle$; see Figure 9. Note that $\gamma$ is unlikely to be an integer. If $\gamma$ is 


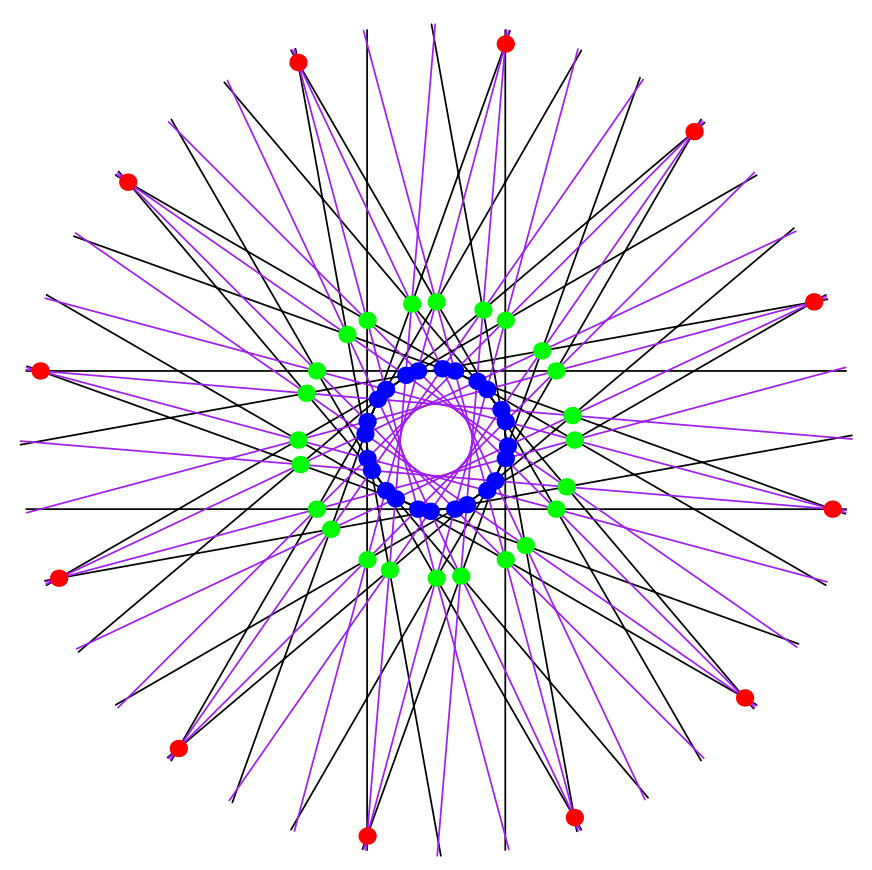

Figure 8: A mixed $[4,5]^{2}$ configuration whose special class of points is farthest away from the center

known, however, then the line $\left\langle v_{\lfloor\gamma\rfloor}, v_{\lfloor\gamma\rfloor+c}\right\rangle$ will intersect point $p_{1}$.

To determine $\gamma$, let $C$ be the midpoint of the line $\left\langle v_{\gamma}, v_{\gamma+c}\right\rangle$, and let $O$ be the center of the circle. Since the total angle from $v_{0}$ to $v_{a}$ is $\frac{2 \pi a}{m}$, a little elementary geometry and trigonometry shows that

$$
A=\cos \left(\frac{a \pi}{m}\right)\left(\cos \left(\frac{2 a \pi}{m}\right), \sin \frac{2 a \pi}{m}\right)
$$

and similarly,

$$
C=\cos \left(\frac{c \pi}{m}\right)\left(\cos \left(\frac{\pi}{m}(2 \gamma+c)\right), \sin \frac{2 \pi}{m}(2 \gamma+c)\right)
$$

Determining when $A$ is on the line $\left\langle v_{\gamma}, v_{\gamma+c}\right\rangle$ is equivalent to determining when the point $A$ lies on the halfplane with normal $C$, i.e., solving

$$
C \cdot C=C \cdot A
$$

Note that $0<a, c, \gamma<m$. Then, equation 3 implies $\left(\right.$ since $\left.\cos ^{2}(\theta)+\sin ^{2}(\theta)=1\right)$ : 


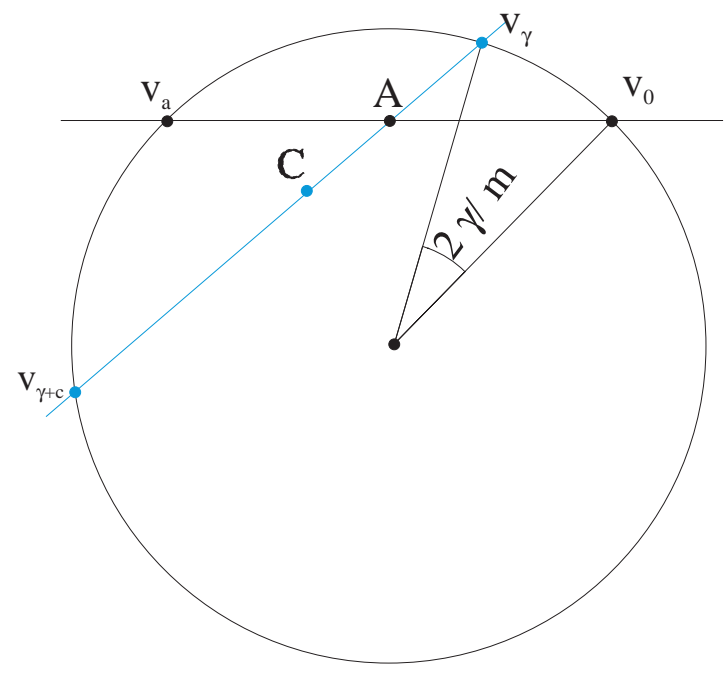

Figure 9: To determine $\gamma$ for mixed $[4,5]^{2}$ configurations

$$
\begin{aligned}
\cos ^{2}\left(\frac{c \pi}{m}\right)= & \cos \left(\frac{c \pi}{m}\right) \cos \left(\frac{a \pi}{m}\right)\left(\cos \left(\frac{a \pi}{m}\right) \cos \left((2 \gamma+c) \frac{\pi}{m}\right)\right. \\
& \left.+\sin \left(\frac{a \pi}{m}\right) \sin \left((2 \gamma+c) \frac{\pi}{m}\right)\right) \\
\frac{\cos \left(\frac{c \pi}{m}\right)}{\cos \left(\frac{a \pi}{m}\right)}= & \cos \left((a-(2 \gamma+c)) \frac{\pi}{m}\right) \\
\arccos \left(\frac{\cos \left(\frac{c \pi}{m}\right)}{\cos \left(\frac{a \pi}{m}\right)}\right)= & (a-2 \gamma-c) \frac{\pi}{m} \\
\gamma & =\frac{m\left(c-a+\arccos \left(\frac{\cos \left(\frac{c \pi}{m}\right)}{\cos \left(\frac{a \pi}{m}\right)}\right)\right)}{2 \pi}
\end{aligned}
$$

Choose the $t^{t h}$ intersection point $p_{t}$, and note that it lies on the $c$-line $\left\langle v_{t+\lfloor\gamma\rfloor}, v_{t+\lfloor\gamma\rfloor+c}\right\rangle$. For the following, it may be helpful to refer to Figure 10. Let $d=d\left(O, p_{t}\right)$ be the distance from the origin to $p_{t}$. Let $\alpha$ be the angle from the midpoint of the span $a$ diagonal, which will now be called $M_{a}$, to point $p_{t}$ and let $\beta$ be the angle from the midpoint of the span $c$ diagonal, $M_{c}$, which also passes through $p_{t}$ to point $p_{t}$. Note that

$$
d=\frac{\cos \left(\frac{a \pi}{m}\right)}{\cos (\alpha)} \text { and } d=\frac{\cos \left(\frac{b \pi}{m}\right)}{\cos (\beta)}
$$

Also,

$$
\alpha+\beta+\frac{\pi a}{m}=\frac{2 \pi}{m}(\lfloor\gamma\rfloor+t)+\frac{c \pi}{m}
$$




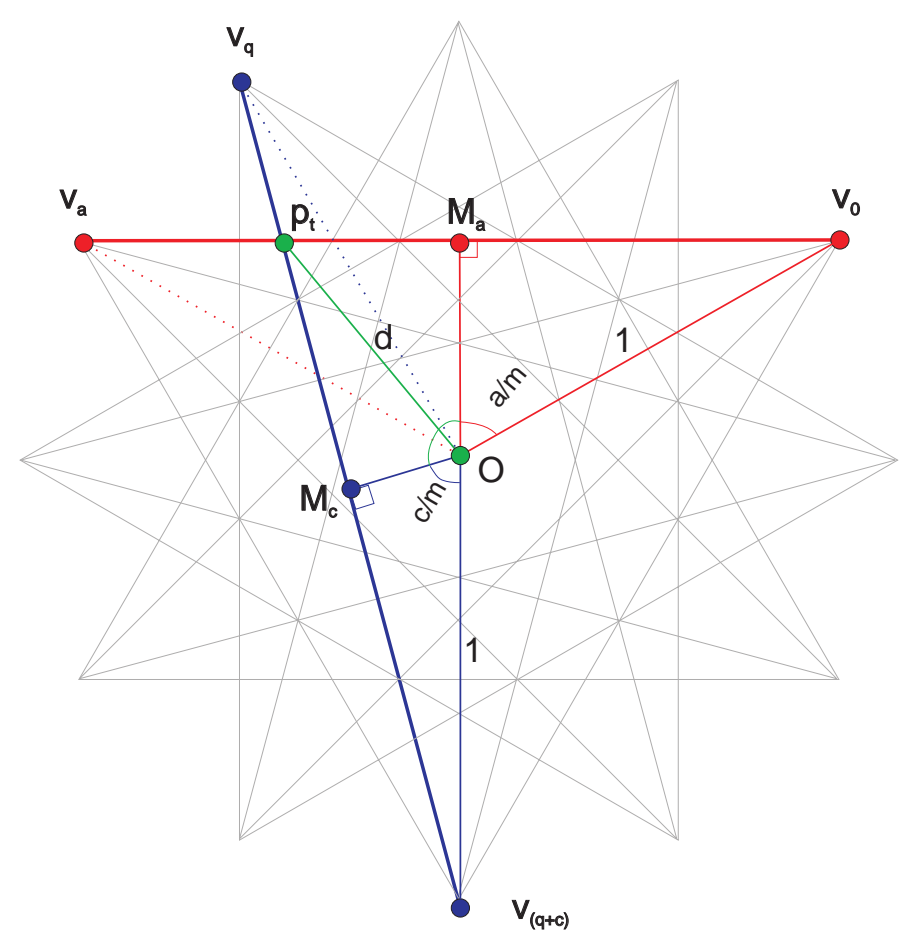

Figure 10: Determining $\alpha$ and $\beta$ to construct a mixed configuration. Note $q=t+\lfloor\gamma\rfloor$.

since that measures the distance from $v_{0}$ to the midpoint of $\left\langle v_{t+\lfloor\gamma\rfloor}, v_{t+\lfloor\gamma\rfloor+c}\right\rangle$ in two ways. Therefore,

$$
\beta=\frac{\pi(c-a+2(t+\lfloor\gamma\rfloor))}{m}-\alpha
$$

For convenience, let

$$
z=\frac{\pi(c-a+2(t+\lfloor\gamma\rfloor))}{m}
$$

Note that $d\left(O, M_{a}\right)=\cos \left(\frac{\pi a}{m}\right)$ and $d\left(O, M_{c}\right)=\cos \left(\frac{\pi c}{m}\right)$. Using elementary righttriangle trigonometry,

$$
\begin{aligned}
d\left(O, p_{t}\right) & =\frac{d\left(O, M_{a}\right)}{\cos (\alpha)}=\frac{d\left(O, M_{c}\right)}{\cos (\beta)} \\
\frac{\cos (\beta)}{\cos (\alpha)} & =\frac{\cos (z-\alpha)}{\cos (\alpha)}=\frac{\cos \left(\frac{\pi c}{m}\right)}{\cos \left(\frac{\pi a}{m}\right)} \\
\frac{\cos z \cos \alpha+\sin z \sin \alpha}{\cos \alpha} & =\frac{\cos \left(\frac{\pi c}{m}\right)}{\cos \left(\frac{\pi a}{m}\right)} \\
\cos z+\sin z \tan \alpha & =\frac{\cos \left(\frac{\pi c}{m}\right)}{\cos \left(\frac{\pi a}{m}\right)}
\end{aligned}
$$


Hence,

$$
\alpha=\arctan \left(\frac{\cos \left(\frac{\pi c}{m}\right)}{\cos \left(\frac{\pi a}{m}\right) \sin \left(\frac{\pi(c-a+2(t+\lfloor\gamma\rfloor))}{m}\right)}-\cot \left(\frac{\pi(c-a+2(t+\lfloor\gamma\rfloor))}{m}\right)\right)
$$

Therefore, given a particular choice of $a-c$ intersection in a $[4,4]^{1}$ configuration, it is possible to construct explicitly the corresponding mixed astral $[4,5]^{2}$ configuration; Figure 7 shows such a configuration.

Note that none of the mixed $[4,5]^{2}$ configurations have lines passing through the center of the configuration, so their polars are astral $[5,4]^{2}$ configurations; for convenience, these will also be known as mixed configurations. For example, see Figure 11.

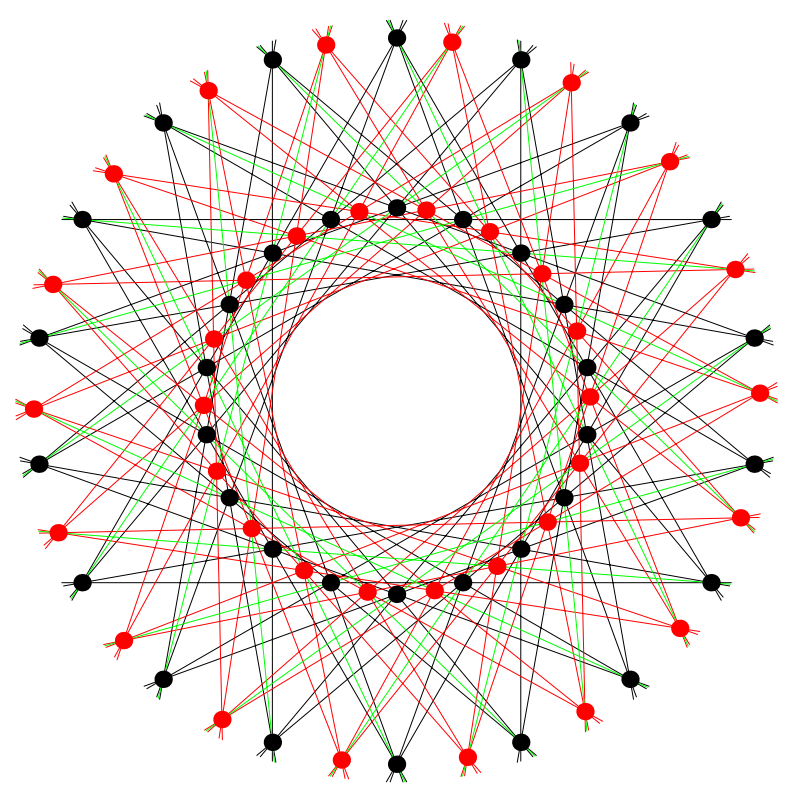

Figure 11: A mixed astral $[5,4]^{2}$ configuration; it is the polar of the configuration in Figure 7.

\section{$7 \quad$ Other astral configurations}

\subsection{Astral $[6,5]$ configurations}

It has been shown, in Corollary 5.3, that there are no astral $[6,5]^{1}$ configurations. Lemma 6.2 says that the only possible astral $[6,5]^{2}$ configurations are ordinary and mixed; however, since there are no $[6,5]^{1}$ configurations, there can be no ordinary type 2 configurations, either. The discussion preceding Lemma 6.2 shows that to construct a mixed 
$[6,5]^{2}$ configuration, it is necessary to have a $[6,4]^{1}$ configuration with a 3 -embryonic point. Such configurations do exist; for example, see Figures 12 and 13. In [12], B. Poonen and M. Rubinstein characterize all intersections of three diagonals of a regular polygon which are internal to that polygon.

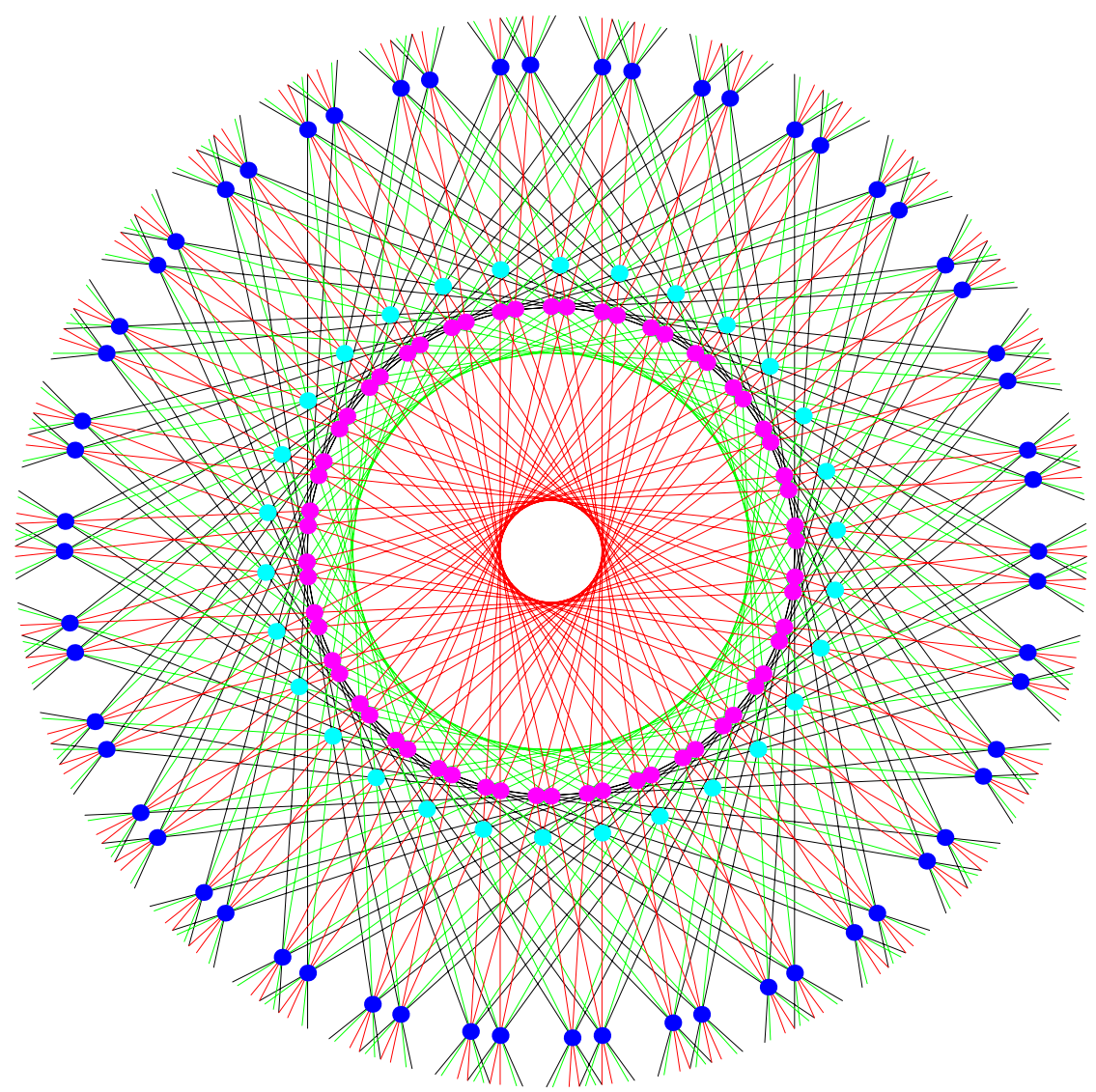

Figure 12: A mixed astral $[6,5]^{2}$ configuration, formed from two copies of the $[6,4]^{1}$ configuration $30 \# 10_{1} 11_{6} 14_{13}$.

Recall that no $s$-embryonic point lies on a line of symmetry of the astral $[q, k]^{1}$ configuration which contains it. Thus, to determine whether a 3-diagonal intersection listed in [12] may be 3-embryonic, it suffices to determine whether the angle between the point and horizontal is an integral multiple of $\frac{\pi}{m}$ for $m$ the size of the $[6,4]^{1}$ configuration. Theorem 7.1. The only mixed astral $[6,5]^{2}$ configurations whose special class of points lie closer to the center of the configuration than one of the non-special classes of points are formed from the astral $[6,4]^{1}$ configurations $30 \# 8_{1} 10_{7} 13_{12}$ and $30 \# 10_{1} 11_{6} 14_{13}$.

Proof. In [12], Poonen and Rubinstein show that if points $A, B, C, D, E, F$ are on the circle with arclengths $u, x, v, y, w, z$, respectively, between successive points, then the three diagonals formed by chords $A D, B E, C F$ intersect precisely when 


$$
\begin{aligned}
\sin (\pi U) \sin (\pi V) \sin (\pi W) & =\sin (\pi X) \sin (\pi Y) \sin (\pi Z) \\
U+V+W+X+Y+Z & =1
\end{aligned}
$$

where $U=u /(2 \pi)$, etc. [12, equation (2.2)]. Furthermore, they show that there are three different types of solutions to the above equations:

1. Trivial solutions, where $U+V+W=1 / 2$ and $X, Y, Z$ are a permutation of $U, V, W$;

2. Four 1-parameter families of solutions;

3. 65 sporadic solutions.

To match the notation of [12] with the notation of $[6,4]$ configurations, notice that if $a, c, z$ are the spans of the diagonals, then

$$
\begin{gathered}
a=2 m(U+X+V) \\
m-a=2 m(Y+W+Z) \\
c=2 m(X+V+Y) \\
m-c=2 m(W+Z+U) \\
z=2 m(V+Y+W) \\
m-z=2 m(Z+U+X)
\end{gathered}
$$

Let $\bar{U}=2 m U$, etc. Since we are looking for mixed $[6,5]^{2}$ configurations to be formed from $[6,4]^{1}$ configurations, in particular, we are interested in $m$-gons where $m$ is divisible by 30 . Consider the possible trivial solutions. Suppose there is a trivial solution associated with $m=30 q$ which has spans which correspond to those which generate a $[6,4]^{1}$ configuration. Previously, it has been shown that $q$ divides the span, since any $[6,4]^{1}$ configuration with $m=30 q$ is constructed using $q$ copies of the astral configuration with $m=30$. The trivial solution must be of the form $\bar{U}+\bar{V}+\bar{W}=\frac{30 q}{2}$, with $\bar{X}, \bar{Y}, \bar{Z}$ a permutation of $\bar{U}, \bar{V}, \bar{W}$. Using this fact, equations 9 - 14 listed above, and the elementary number-theoretic result that if $\alpha \mid(\beta+\gamma)$ and $\alpha \mid \gamma$ then $\alpha \mid \beta$, it is straightforward to show that $q$ divides each of $\bar{U}, \bar{V}, \bar{W}, \bar{X}, \bar{Y}, \bar{Z}$.

Thus, any trivial solution for $m=30 q$ must be a trivial solution for $m=30$ multiplied by $q$. Hence, it suffices to determine the 3 -embryonic points associated with trivial solutions for $m=30$, those which arise from the sporadic solutions whose common denominator is divisible by 30 (specifically, 30,60, 90,120, and 210), and those which arise from the one-parameter families. This was accomplished by writing computer programs which, when given a sequence $(U, V, W, X, Y, Z)$, calculated the spans and the angle between the point and horizontal. Those sequences which had angles which were not integral multiples 
of $\frac{\pi}{m}$ were selected and their spans were considered to see if the spans formed an astral $[6,4]$ configuration.

To compute the trivial sequences, all compositions of 15 into three non-zero parts were found and they were joined with all permutations of the compositions found. After selecting for angles which were non-integral multiples of $\frac{\pi}{m}$ and points other than the center and looking for acceptable spans, four sequences were found, all from the trivial solutions.

$$
\begin{aligned}
& (\bar{U}, \bar{V}, \bar{W}, \bar{X}, \bar{Y}, \bar{Z})=(1,6,8,1,6,8) \text { in } 30 \# 8_{1} 10_{7} 13_{12}, \\
& (\bar{U}, \bar{V}, \bar{W}, \bar{X}, \bar{Y}, \bar{Z})=(1,6,8,6,8,1) \text { in } 30 \# 8_{1} 10_{7} 13_{12}, \\
& (\bar{U}, \bar{V}, \bar{W}, \bar{X}, \bar{Y}, \bar{Z})=(2,6,7,2,6,7) \text { in } 30 \# 10_{1} 11_{6} 14_{13}, \\
& (\bar{U}, \bar{V}, \bar{W}, \bar{X}, \bar{Y}, \bar{Z})=(2,6,7,6,7,2) \text { in } 30 \# 10_{1} 11_{6} 14_{13} .
\end{aligned}
$$

Note the point determined by sequence $(1,6,8,6,8,1)$ is a rotation of the point determined from $(1,6,8,1,6,8)$, and similarly for $(2,6,7,2,6,7)$ and $(2,6,7,6,7,2)$.

Checking the sporadic sequences was tedious but easy. Checking the families was more complicated. It was necessary to change notation by setting $t=\frac{j}{30 q}$ to achieve $m=30 q$, noticing that the allowable values for $j$ are $j=q, 2 q, 3 q, 4 q, 5 q$ and then calculating the spans. There was only one sequence with appropriate spans, and this sequence participates in a six-diagonal intersection.

Note that this is not an exhaustive classification of all astral $[6,5]^{2}$ configurations; in particular, Theorem 7.1 does not classify the $[6,5]^{2}$ configurations whose special class of points is the farthest symmetry class from the center of the configuration. (For an example of such a configuration, see Figure 13.) To classify these configurations, the notion of isogonal conjugation is useful.

\subsection{Isogonal conjugation}

Suppose a triangle $A B C$ has angle bisectors $\alpha$ bisecting $\angle A, \beta$ bisecting $\angle B$, and $\gamma$ bisecting $\angle C$. If $l$ is a line passing through $A$, the isogonal conjugate line $l^{\prime}$ is the reflection of $l$ through $\alpha$ (see Figure 14 (a)). It is a well-known result (e.g., [1]) that given a point $P$ and the lines $P A=l, P B=m$, and $P C=n$, the isogonal conjugate lines $l^{\prime}, m^{\prime}$, and $n^{\prime}$ intersect in a single point $P^{\prime}$, the isogonal conjugate point of $P$ (see Figure $14(\mathrm{c})$ ).

Given a triangle $A B C$ and parallel lines $l$ passing through $A, m$ passing through $B$, and $n$ passing through $C$, it is known that the isogonal conjugate lines $l^{\prime}, m^{\prime}, n^{\prime}$ intersect at a point on the circumcircle of $\triangle A B C$ (Figure $14(\mathrm{~b})$ ). Since the isogonal conjugate lines are constructed by reflecting over the appropriate angle bisectors, changing the angle of lines $l$ and $n$ (assuming that $m$ is between $l$ and $n$ ) so that $l, m$, and $n$ intersect at a point $P$ somewhere outside the circumcircle causes the isogonal conjugate point $P^{\prime}$ to move interior to the circumcircle, since the change of angle is a continuous function (Figure 14 (c) again). Finally, note that isogonal conjugation is an involution. 


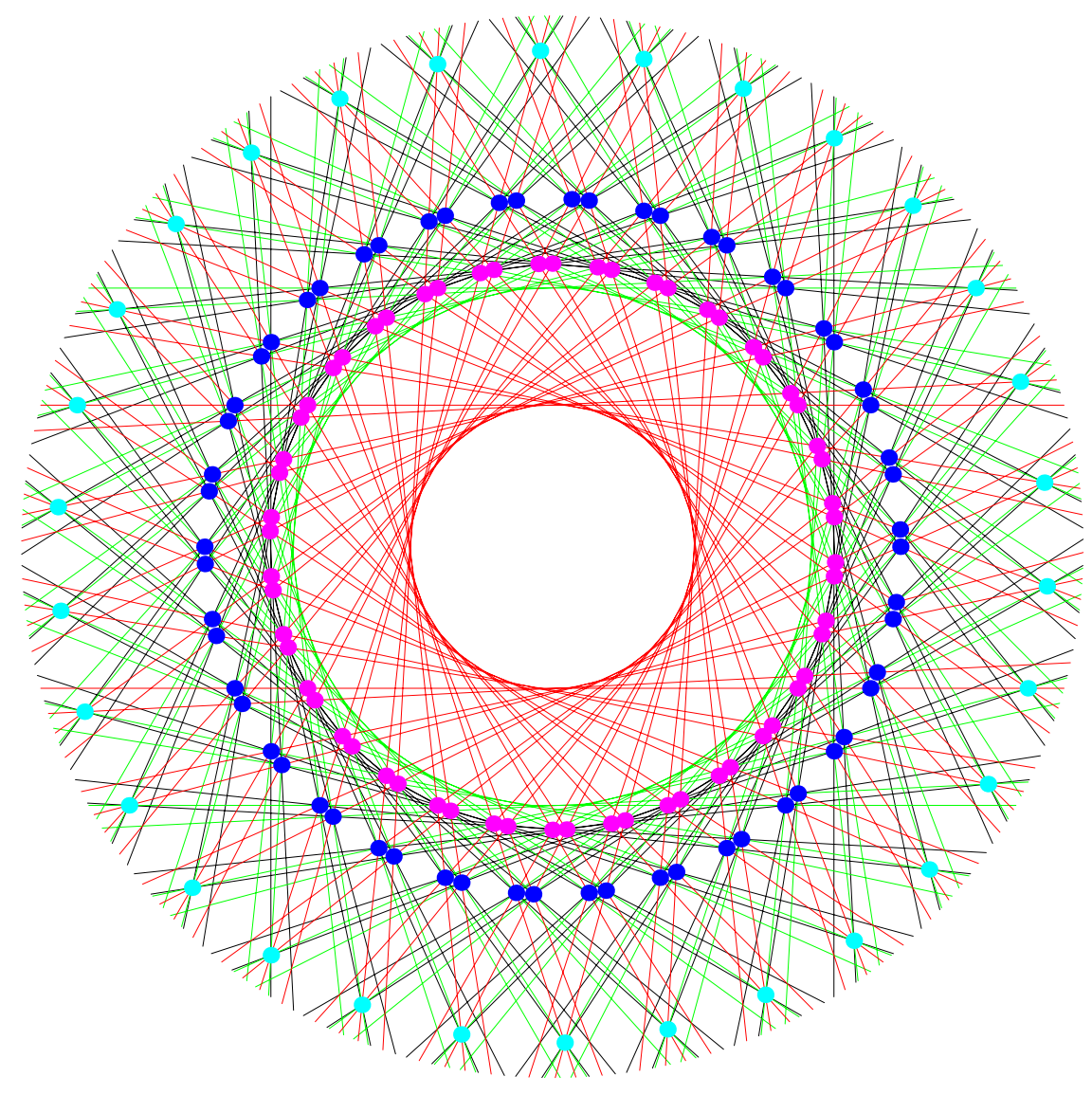

Figure 13: A mixed astral $[6,5]^{2}$ configuration, formed from two copies of the $[6,4]^{1}$ configuration $30 \# 10_{1} 11_{6} 14_{13}$ with its special class of points farthest from the origin.

Corollary 7.2. Astral $[6,5]^{2}$ configurations whose special class of points is farthest from the center of the origin may be mixed using appropriate 3-embryonic points in the astral $[6,4]^{1}$ configurations $30 \# 8_{1} 10_{7} 13_{12}$ and $30 \# 10_{1} 11_{6} 14_{13}$.

Proof. Suppose an external 3-embryonic point $P$ existed in some $[6,4]^{1}$ configuration. Construct its isogonal conjugate with respect to a triangle whose vertices are chosen from the points formed by the intersection of the diagonals with the circle passing through the outer ring of points, with the restriction that for the middle line, the intersection point closer to $P$ is chosen. The isogonal conjugate is an internal 3-embryonic point. Moreover, since isogonal conjugation is an involution, all external 3-embryonic points must be (appropriate) isogonal conjugates of internal 3-embryonic points. By Theorem 7.1, all internal 3-embryonic points in any astral [6,4] configuration have been classified. Thus, astral $[6,5]^{2}$ configurations whose special class of points is farthest from the center of the origin may be mixed using the isogonal conjugates of the 3-embryonic points discussed in the proof of Theorem 7.1. 


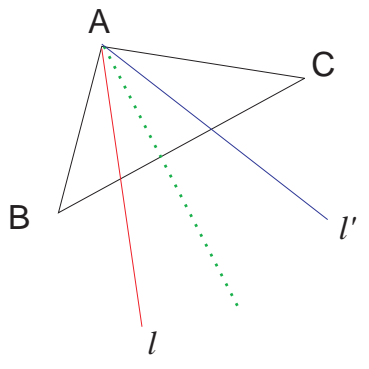

(a)

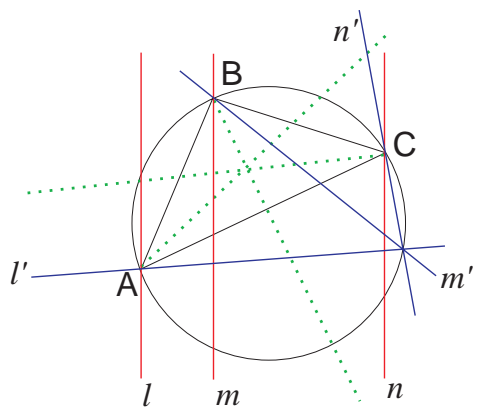

(b)

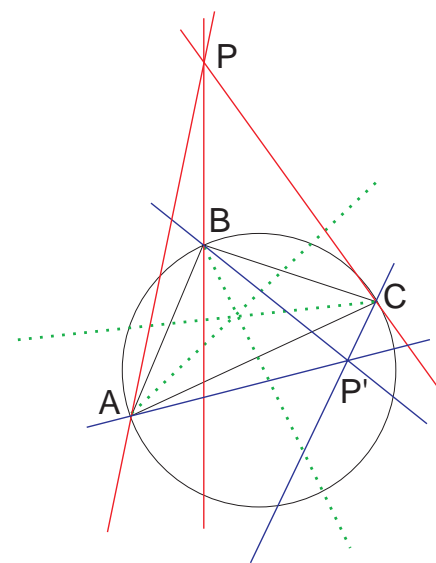

(c)

Figure 14: Isogonal conjugation. (a) A line and its isogonal conjugate line. (b) A triangle with its circumcircle and a set of three parallel lines, whose isogonal conjugate lines intersect at a single point on the circumcircle. (c) Given a point $P$ outside a circle which is coincident with three lines, its isogonal conjugate point lies inside the circle.

\subsection{Astral $[5,6]$ configurations}

The mixed configurations discussed in subsection 7.1 have polars in the Euclidean plane which are astral $[5,6]^{2}$ configurations, since none of the mixed $[6,5]^{2}$ configurations contain diameters.

In addition, there are astral $[5,6]^{1}$ configurations formed by adding diameters to $[4,6]^{1}$ configurations, denoted as $m \#\left(a_{b} c_{d}\right)\left(a_{e} c_{f}\right), D$. By looking at the list of astral $[4,6]^{1}$ configurations and applying Lemma 2.2, diameters may be added to any even multiple of a $[4,6]$ configuration and to no others. These type 1 configurations also may be used to generate ordinary astral $[5,6]^{2}$ configurations.

There are no other $[5,6]^{1}$ configurations since there are no astral $[6,6]$ configurations from which to construct them, by Lemma 4.2 .

\subsection{Astral $[7,4]$ and $[4,7]$ configurations}

By Theorem 3.6 there are no astral $[4,8]^{1}$ configurations from which half the lines of a symmetry class could be removed, so the only astral $[7,4]^{1}$ configurations are those formed by adding diameters to astral $[6,4]^{1}$ configurations, denoted as $m \# a_{b} c_{d} e_{f}, D$. By inspecting the list of $[6,4]^{1}$ configurations and applying Lemma 2.2 , it is possible to add diameters to any even multiple of any $[6,4]$ configuration and to any multiple of the configuration $30 \# 9_{3} \quad 10_{6} 12_{10}$. A $[7,4]^{1}$ configuration constructed in this fashion using $30 \# 9_{3} \quad 10_{6} \quad 12_{10}$ is displayed in Figure 15 . Ordinary $[7,4]^{2}$ configurations may be constructed, as usual, using the $[7,4]^{1}$ configurations. 


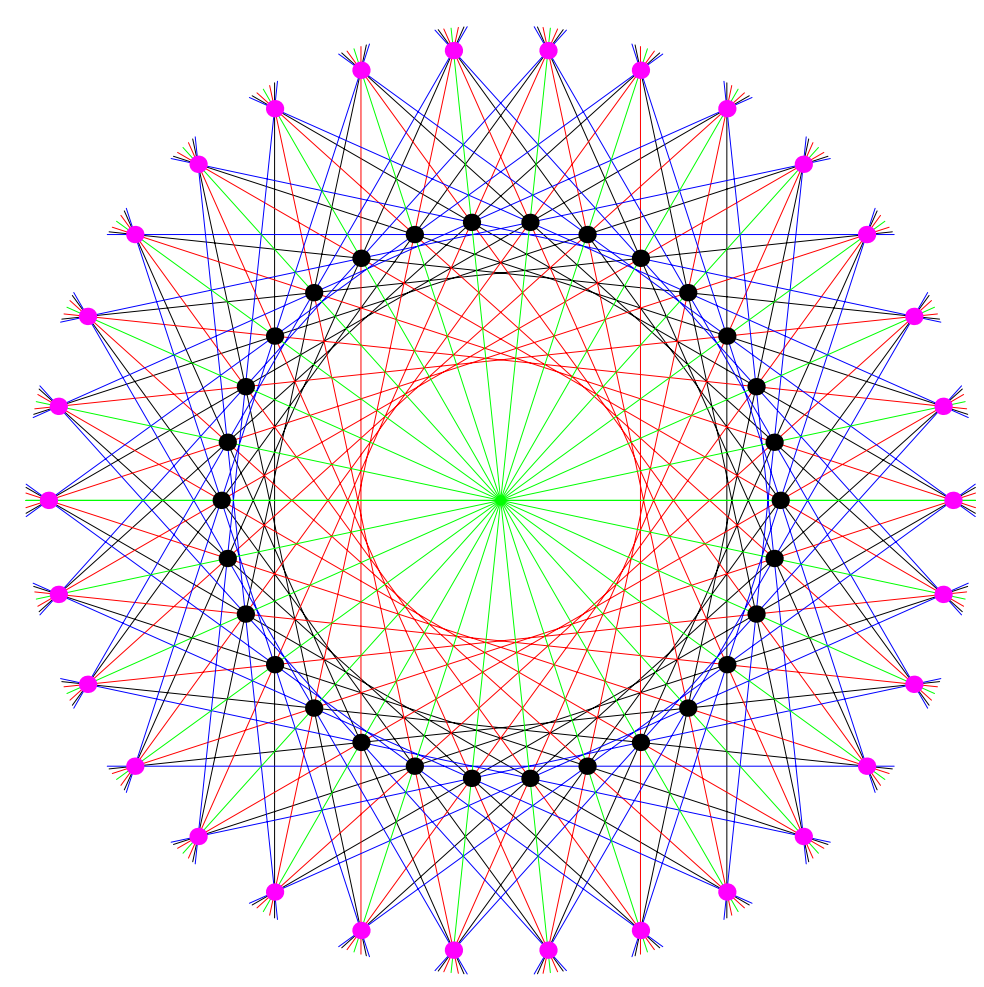

Figure 15: The astral $[7,4]^{1}$ configuration formed by adding diameters to the $[6,4]$ configuration $30 \# 10_{6} 12_{10} 9_{3}$.

There are no astral $[4,7]^{1}$ configurations: to see this, recall from Theorem 3.6 that there are no $[4,8]^{1}$ configurations, and apply Lemma 4.1 with $t=3$ and $s=2$. Starting with a $[4,6]^{1}$ configuration, it is easy to construct mixed $[4,7]^{2}$ configurations; use the same embryonic points and construction methods which were used to construct mixed $[4,5]^{2}$ configurations (see section 6.2 ). To see that this yields $[4,7]^{2}$ configurations, note that the new points are $a-c$ embryonic points, and so after rotation each will have precisely 4 lines passing through it, while each line will now have 7 points on it.

Since none of the lines of the mixed $[4,7]^{2}$ configurations are diameters, the polars of these configurations will be $[7,4]^{2}$ configurations; for example, see Figure 17.

\section{Some results on $[5,5]$ configurations}

Recall that every $[4,5]^{1}$ configuration is constructed by taking every other point from a suitable symmetry class of points in the appropriate $[4,6]^{1}$ configuration. Suppose that a $[5,5]^{1}$ configuration could be constructed by adding diameters to a $[4,5]^{1}$ configuration. Each diameter must pass through a point in the special class of points. It follows that 


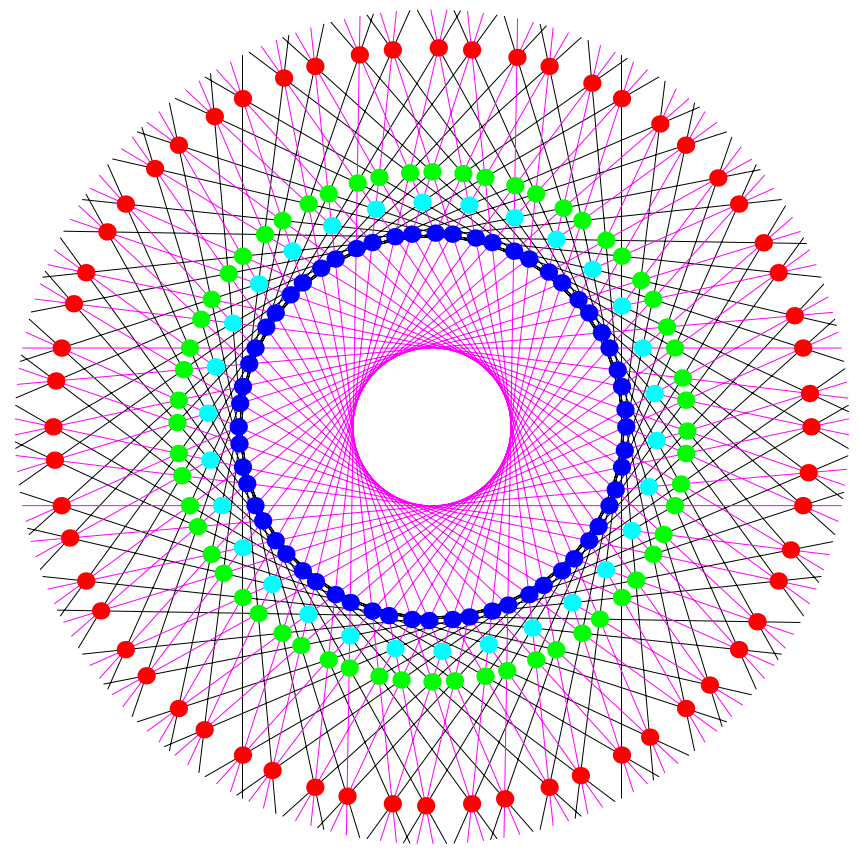

Figure 16: A mixed astral $[4,7]^{2}$ configuration, formed from two copies of the configuration $30 \#\left(10_{2} 13_{11}\right)\left(10_{7} 13_{12}\right)$.

$m \equiv 2 \bmod 4$, since if $m \equiv 0 \bmod 4$ then some diameters would pass through two special points and some would pass through none. In fact, each special point in the $[5,5]^{1}$ configuration would have to be "mirrored" by an unchosen point, so that the existence of a $[5,5]^{1}$ configuration which uses diameters implies the existence of a $[4,5]^{1}$ configuration where the diameters pass through points in each of the three symmetry classes of points.

Proposition 8.1. There are no astral $[5,5]^{1}$ configurations.

Proof. Case 1: An astral $[5,5]^{1}$ configuration is constructed by adding diameters to a $[4,5]^{1}$ configuration. The only astral $[4,5]^{1}$ configurations which could have diameters added to them are $(30 q) \#\left((10 q)_{(6 q)}(12 q)_{(10 q)}\right)\left((10 q)_{(3 q)}(12 q)_{(9 q)}\right) *$ where $q$ is odd (so that $30 q \equiv 2 \bmod 4)$; but the diameters would not pass through the points with symbol $(10 q)_{(3 q)}$ since by Lemma 2.2 the parity is wrong.

Case 2: The astral $[5,5]^{1}$ configuration is constructed by removing half the lines from a symmetry class of an appropriate $[6,5]^{1}$ configuration. However, according to Corollary 5.3, there are no $[6,5]^{1}$ configurations.

It follows that there are no ordinary $[5,5]^{2}$ configurations, either. So, if an astral $[5,5]^{2}$ configuration does exist, it must be constructed as a mixed configuration. Either it is mixed from a $[5,4]^{1}$ configuration or it is even more complicated, with an additional 


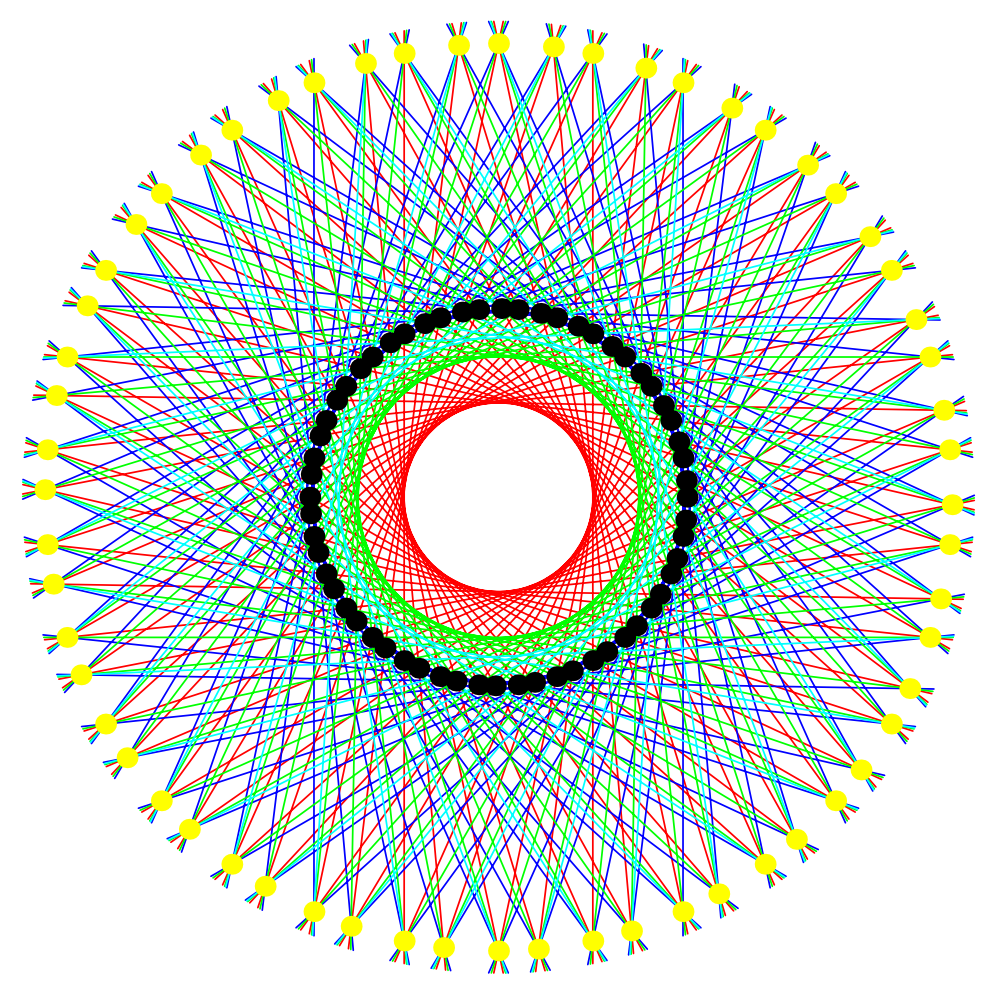

Figure 17: A mixed astral $[7,4]^{2}$ configuration, the polar of the configuration in Figure 16.

special class of points and an additional special class of lines added to an ordinary $[4,4]^{2}$ configuration. Such a configuration will be called twice-mixed.

Proposition 8.2. There are no astral $[5,5]^{2}$ configurations mixed from two $[5,4]^{1}$ configurations.

Proof. Suppose the proposition is false. To be constructed as a mixed configuration, there must be a 3-embryonic point which is the intersection point of exactly one line of each of three spans. Note that in a $[5,4]^{1}$ configuration which uses diameters, there are no 3 -embryonic points. Thus, the $[5,5]^{2}$ configuration would have to be mixed from the configuration $(30 q) \#(10 q)_{(6 q)}(12 q)_{(10 q)}(9 q)_{(3 q)} *$, constructed by removing half of one of the symmetry classes of lines from the $[6,4]$ configuration $(30 q) \#(10 q)_{(6 q)}(12 q)_{(10 q)}(9 q)_{(3 q)}$ (see Section 5.2). Through each point in the configuration $(30 q) \#(10 q)_{(6 q)}(12 q)_{(10 q)}(9 q)_{(3 q)} *$ pass two lines of span 10q, two lines of span 12q, and one line of span $9 q$. In order to be able to construct the mixed configuration, one of the underlying $[5,4]^{1}$ configurations would need to have a 3 -embryonic point (using all three spans, of course), and in the corresponding position on the other underlying configuration, it would need to be a 2embryonic point using the $10 q$ and $12 q$ lines only. If this were the case, then rotating the configurations appropriately and superimposing them as usual would lead to a point 
with the correct number of lines passing through it. In particular, one needs the $[6,4]$ configuration $(30 q) \#(10 q)_{(6 q)}(12 q)_{(10 q)}(9 q)_{(3 q)}$ to contain 3-embryonic points. The same computer calculations which determined the possible mixed $[6,5]^{2}$ configurations shows that there are no 3-embryonic points inside the circle which passes through the points with symbol $9_{9}=10_{10}=12_{12}$; these will be called internal 3 -embryonic points. Thus , to show that there are no astral $[5,5]^{2}$ configurations mixed from two $[5,4]^{1}$ configurations, it remains to show that there are no external 3-embryonic points in the configuration $(30 q) \#(10 q)_{(6 q)}(12 q)_{(10 q)}(9 q)_{(3 q)}$. For convenience in the subsequent argument, suppose that this circle is chosen to be the unit circle.

It follows that if a 3-embryonic point $P$ existed in the $[6,4]$ configuration $(30 q) \#(10 q)_{(6 q)}(12 q)_{(10 q)}(9 q)_{(3 q)}$ that was exterior to the unit circle, then its isogonal conjugate point (with respect to a triangle whose vertices are chosen from the points formed by the intersection of the diagonals with the circle with the restriction that for the middle line, the intersection point closer to $P$ is chosen, say) will exist and be an internal 3-embryonic point. But no such point exists, so no external 3-embryonic point can exist either.

Thus, the only possible remaining candidate for an astral $[5,5]$ configuration is if it is possible to construct one from two $[4,4]$ configurations, i.e., by starting with a mixed $[4,5]^{2}$ configuration and adding a new, special class of lines (this is equivalent to constructing a twice-mixed configuration). Note that to ensure that every point would only have 5 lines passing through it, the new class of lines would have to be perpendicular to the rays from the center of the configuration which pass through the special class of points. While no examples have been found of configurations which are constructed in this fashion, so far an argument showing that no such configurations exist has not been found.

Conjecture 1. There are no astral [5,5] configurations.

\section{Astral $[7,5]$ and $[5,7]$ configurations.}

Since there are no $[4,7]^{1}$ configurations, it is impossible to construct a $[5,7]^{2}$ configuration mixed from two $[4,7]^{1}$ configurations. Moreover, beginning with a $[7,4]^{1}$ configuration and trying to mix a $[7,5]^{2}$ configuration by adding a special class of points fails also, because the special class of points would have to be formed from an $a-c$ embryonic point, none of which lie on a diameter, and so the special class of lines, which are diameters, would not pass through the new points. Hence no such configuration can exist.

The lack of $[6,5]^{1}$ configurations implies the lack of type 1 and ordinary type $2[7,5]$ configurations, by Lemma 3.7. Also, there are no astral $[7,5]^{2}$ configurations formed by mixing two $[7,4]^{1}$ configurations, since the only $[7,4]^{1}$ configurations contain diameters and diameters don't pass through embryonic points. Similarly, the lack of $[4,7]^{1}$ configurations implies the lack of $[5,7]^{1}$ configurations and ordinary $[5,7]^{2}$ configurations, by Lemma 3.7. 
There might be twice-mixed $[7,5]^{2}$ or $[5,7]^{2}$ configurations formed from adding a special class of points and a special class of lines to two $[6,4]^{1}$ or $[4,6]^{1}$ configurations, respectively; their existence or nonexistence will be determined when it is known whether there are twice mixed $[5,5]$ configurations, since the twice mixed $[7,5]$ and $[5,7]$ configurations may be constructed by adding a non-special class of lines or points, respectively, to a twice mixed $[5,5]$ configuration.

Conjecture 2. There are no astral $[7,5]$ and $[5,7]$ configurations.

\section{Summary of Configuration Notation}

Table 2: Notation for type 1 astral configurations

\begin{tabular}{|c|c|c|}
\hline$[q, k]$ configuration & Notation & comments \\
\hline$[4,4]^{1}$ & $m \# a_{b} c_{d}$ & $a_{b}=c_{d}$ \\
{$[6,4]^{1}$} & $m \# a_{b} c_{d} z_{w}$ & $a_{b}=c_{d}=z_{w}$ \\
{$[4,6]^{1}$} & $m \#\left(a_{b} c_{d}\right)\left(a_{e} c_{f}\right)$ & $a_{b}=c_{d}$ and $a_{e}=c_{f}$ \\
{$[4,5]^{1}$} & $m \#\left(a_{b} c_{d}\right)\left(a_{e} c_{f}\right)^{*}$ & Uses half the $a_{e}$ points \\
{$[5,4]^{1}$} & $m \# a_{b} c_{d} e_{f}^{*}$ & Uses half the $a_{b}$ points \\
{$[5,4]^{1}$} & $m \# a_{b} c_{d}, D$ & includes diameters \\
{$[5,6]^{1}$} & $m \#\left(a_{b} c_{d}\right)\left(a_{e} c_{f}\right), D$ & includes diameters \\
{$[7,4]^{1}$} & $m \# a_{b} c_{d} e_{f}, D$ & includes diameters \\
\hline
\end{tabular}

Table 2 summarizes the different notation for various type 1 astral configurations. Type 2 configurations either are constructed using two copies of these (ordinary type 2) or as mixed configurations. The method of constructing mixed configurations was discussed extensively, but there is no specific notation for them.

\section{Open Questions}

The case of astral $[3,3]$ configurations is quite different than the case of astral $[q, k]$ configurations when $q$ and $k$ are at least four. For example, a single discrete symbol describing a configuration may correspond to more than one [3,3] configuration. In addition, although all $[q, k]$ configurations with $q, k \geq 4$ have dihedral symmetry, there exist astral [3,3] configurations with only cyclic symmetry. For an example of these, see Figure 18; note that both configurations pictured correspond to the same discrete parameters. Finally, some astral $n_{3}$ configurations come in continuous families; part of one such family is shown in Figure 19. 
B. Grünbaum presents some results on astral $[3,3]$ configurations with only cyclic symmetry in $[11, \S 26]$, where a single discrete symbol usually corresponds to a pair of polar configurations; the example in Figure 18 shows such a pair. Additionally, [3,3] configurations were discussed in [7]. I have a few results on astral [3,3] configurations with dihedral symmetry (see Figure 19): in this case, a single discrete symbol usually correspond to a continuous family of configurations, where a single parameter may vary continuously. (One way of viewing the continuous parameter is that it measures the angle between two vertices in the same symmetry class, where one vertex is the reflection in the horizontal of the other; in Figure 19, the continuous parameter measures then angle between a pair of blue (inner) points and varies between 0 and $\frac{2 \pi}{5}$ in increments of $\frac{\pi}{5}$.)
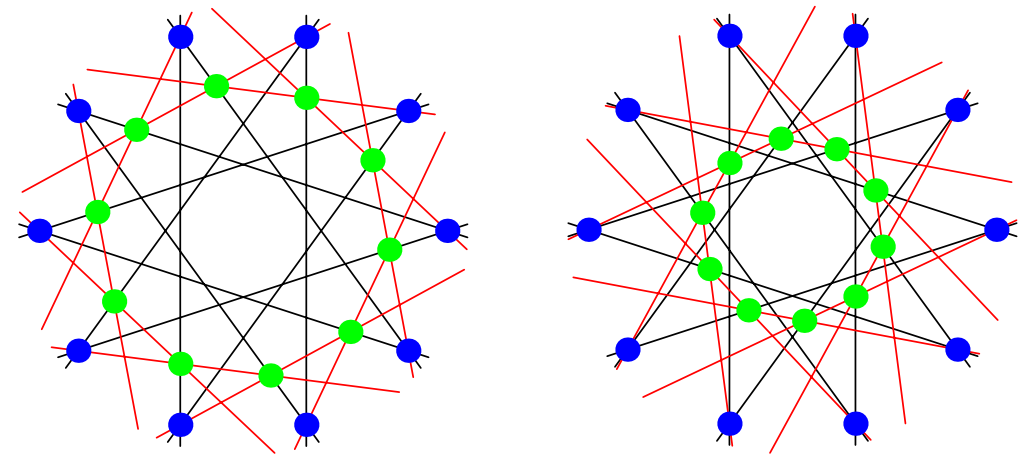

Figure 18: Two $[3,3]$ configurations with cyclic symmetry
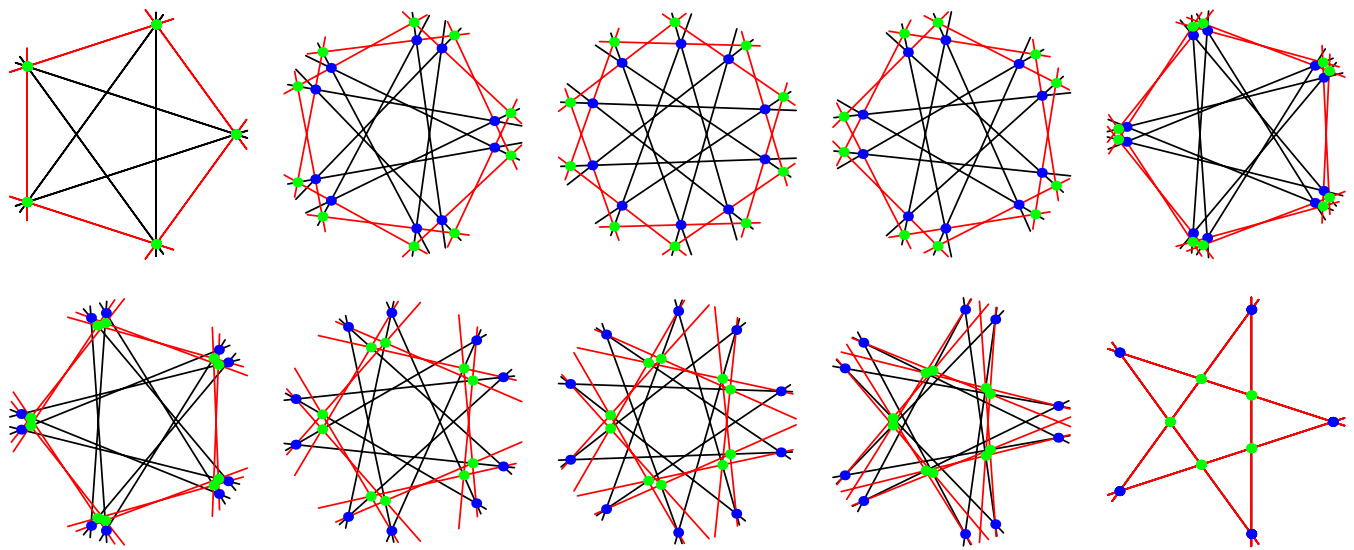

Figure 19: Members of a continuous family of astral [3,3] configurations with dihedral symmetry. (Note that the points and lines of the configuration are not always distinct.)

The question of astral $[3, k]$ and $[k, 3]$ configurations, where $k>3$, appears to be almost totally open; a few results are discussed in [11, §§17-19]. 


\section{References}

[1] Altschiller-Court, N. College geometry: an introduction to the modern geometry of the triangle and the circle. Barnes \& Noble, (1952) $267-274$.

[2] Berman, L.W. Even astral configurations. The Electronic Journal of Combinatorics 11 (2004) \#R37

[3] Berman, L.W. A characterization of astral $\left(n_{4}\right)$ configurations. Discrete and Computational Geometry. 264 (2001), 603 - 612.

[4] Berman, L.W. Astral Configurations. Ph.D. Thesis, University of Washington. (2002)

[5] Betten, A., Brinkmann, G., Pisanski, T. Counting symmetric configurations $v_{3}$. Discrete and Applied Mathematics 99 (2000), 331 - 338.

[6] Boben, M. and T. Pisanski, Polycyclic Configurations. European Journal of Combinatorics. 244 (2003) 431-457.

[7] Boben, M., T. Pisanski, and A. Žitnik, I-graphs and the corresponding configurations. Journal of Combinatorial Designs, 136 (2005), 406-424.

[8] Gropp, H. Configurations and their realization. Discrete Mathematics 174 (1997), $137-151$.

[9] Grünbaum, B. Astral $\left(n_{k}\right)$ configurations. Geombinatorics 3 (1993), 32 - 37.

[10] Grünbaum, B. Astral $\left(n_{4}\right)$ configurations. Geombinatorics 9 (2000), 127 - 134.

[11] Grünbaum, B. Configurations. Unpublished manuscript/class notes. Spring 2004.

[12] Poonen, B. and M. Rubinstein. The number of intersection points made by the diagonals of a regular polygon. SIAM J. Discrete Math. 11 (1998), 135 - 156.

[13] Sturmfels, B. and N. White. All $11_{3}$ and $12_{3}$ configurations are rational. Aequationes Mathematicae 39 (1990) $254-260$. 